\title{
DIVISORIAL ZARISKI DECOMPOSITIONS ON COMPACT COMPLEX MANIFOLDS
}

\author{
By SÉBASTIEN BOUCKSOM
}

ABSTRACT. - Using currents with minimal singularities, we introduce pointwise minimal multiplicities for a real pseudo-effective $(1,1)$-cohomology class $\alpha$ on a compact complex manifold $X$, which are the local obstructions to the numerical effectivity of $\alpha$. The negative part of $\alpha$ is then defined as the real effective divisor $N(\alpha)$ whose multiplicity along a prime divisor $D$ is just the generic multiplicity of $\alpha$ along $D$, and we get in that way a divisorial Zariski decomposition of $\alpha$ into the sum of a class $Z(\alpha)$ which is nef in codimension 1 and the class of its negative part $N(\alpha)$, which is an exceptional divisor in the sense that it is very rigidly embedded in $X$. The positive parts $Z(\alpha)$ generate a modified nef cone, and the pseudo-effective cone is shown to be locally polyhedral away from the modified nef cone, with extremal rays generated by exceptional divisors. We then treat the case of a surface and a hyper-Kähler manifold in some detail. Using the intersection form (respectively the Beauville-Bogomolov form), we characterize the modified nef cone and the exceptional divisors. The divisorial Zariski decomposition is orthogonal, and is thus a rational decomposition, which fact accounts for the usual existence statement of a Zariski decomposition on a projective surface, which is thus extended to the hyper-Kähler case. Finally, we explain how the divisorial Zariski decomposition of (the first Chern class of) a big line bundle on a projective manifold can be characterized in terms of the asymptotics of the linear series $|k L|$ as $k \rightarrow \infty$.

\section{(C) 2004 Elsevier SAS}

RÉSUMÉ. - En utilisant des courants à singularités minimales, nous introduisons les multiplicités minimales ponctuelles d'une (1,1)-classe de cohomologie pseudoeffective $\alpha$ sur une variété complexe compacte $X$, qui sont les obstructions locales à l'effectivité numérique de $\alpha$. La partie négative de $\alpha$ est alors définie comme le diviseur effectif réel dont la multiplicité le long d'un diviseur premier $D$ n'est autre que la multiplicité minimale générique de $\alpha$ le long de $D$, et nous obtenons de cette manière une décomposition de Zariski divisorielle de $\alpha$ en la somme d'une classe $Z(\alpha)$ qui est nef en codimension 1 et de la classe de sa partie négative $N(\alpha)$, qui est un diviseur exceptionnel au sens où il est plongé de manière très rigide dans $X$. Les parties positives $Z(\alpha)$ engendrent un cône nef modifé, et nous montrons que le cône pseudoeffectif est localement polyhédral en dehors du cône nef modifié, de rayons extrémaux engendrés par les diviseurs exceptionnels. Nous traitons ensuite le cas d'une surface et d'une variété hyperkählerienne en détail. À l'aide de la forme d'intersection (respectively la forme de Beauville-Bogomolov), nous caractérisons le cône nef modifié et les diviseurs exceptionnels. La décomposition de Zariski divisorielle est orthogonale, et donc rationnelle, ce qui explique le résultat d'existence connu pour les surfaces projectives, que nous étendons ainsi au cas hyperkählérien. Finalement, nous expliquons comment la décomposition de Zariski divisorielle (de la première classe de Chern) d'un fibré en droites gros sur une variété projective peut être caractérisée en terme du comportement asymptotique des systèmes linéaires $|k L|$ lorsque $k \rightarrow \infty$.

(C) 2004 Elsevier SAS 


\section{Introduction}

It is known since the pioneering work of O. Zariski [19] that the study of the ring

$$
R(X, D):=\bigoplus_{k \geqslant 0} H^{0}(X, \mathcal{O}(k D))
$$

where $D$ is an effective divisor on a projective surface $X$, can be reduced to the case where $D$ is numerically effective (nef). The more precise result obtained by Zariski is that any effective Q-divisor $D$ on a projective surface $X$ can be uniquely decomposed into a sum $D=P+N$ where $P$ is a nef Q-divisor (the positive part), $N=\sum a_{j} D_{j}$ is an effective Q-divisor (the negative part) such that the Gram matrix $\left(D_{i} \cdot D_{j}\right)$ is negative definite, and $P$ is orthogonal to $N$ with respect to the intersection form. Zariski shows that the natural inclusion $H^{0}(k P) \rightarrow H^{0}(k D)$ is necessarily an isomorphism in that case, relating the decomposition to the original problem.

The proof of the uniqueness in this decomposition shows that the negative part $N$ only depends on the class $\{D\}$ of $D$ in the Néron-Severi group $N S(X)$, so that $\{D\} \mapsto\{P\}$ yields a map from part of the pseudo-effective cone to the nef cone, which we want to study geometrically.

Building upon the construction by J.-P. Demailly of metrics with minimal singularities on a pseudo-effective line bundle $L$ over a compact complex manifold, we introduce the minimal multiplicity $\nu(\alpha, x)$ of an arbitrary real pseudo-effective $(1,1)$-class $\alpha$ on a compact complex manifold $X$ at some point $x \in X$. This multiplicity $\nu(\alpha, x)$ is the local obstruction at $x$ to the numerical effectivity of $\alpha$. The set of points $x \in X$ at which $\nu(\alpha, x)$ is positive is a countable union of closed analytic subsets which we call the non-nef locus of $\alpha$. It turns out that this nonnef locus contains only finitely many prime divisors (Theorem 3.14), and the divisorial Zariski decomposition of $\alpha$ is then obtained by subtracting from $\alpha$ the divisorial part of its non-nef locus, counting multiplicities. More precisely, we define the negative part of such a class $\alpha$ by setting $N(\alpha)=\sum \nu(\alpha, D) D$, where $D$ ranges over the prime divisors of $X$ and $\nu(\alpha, D)$ is the generic multiplicity of $\alpha$ along $D$ (cf. Section 3). This negative part $N(\alpha)$ is an effective R-divisor which is exceptional in the sense that it is very rigidly imbedded in $X$. For instance, when $X$ is a surface, the divisors we obtain in that way are exactly the effective $\mathbf{R}$-divisors whose support $D_{1}, \ldots, D_{r}$ has negative definite Gram matrix $\left(D_{i} \cdot D_{j}\right)$.

The difference $Z(\alpha):=\alpha-\{N(\alpha)\}$ is a real pseudo-effective $(1,1)$-class on $X$ which we call the Zariski projection of $\alpha$. It is not a nef class, but is somehow nef in codimension 1 , in the sense that its non-nef locus does not contain any prime divisor. The set of such classes is a closed convex cone which we call the modified nef cone. The decomposition $\alpha=Z(\alpha)+\{N(\alpha)\}$ we call the divisorial Zariski decomposition of $\alpha$, and it is just induced by the Siu decomposition of a closed positive current with minimal singularities in $\alpha$ when the latter is big. For such a big class, we give a criterion to recognize a decomposition $\alpha=p+\{N\}$ into a modified nef and big class and the class of an effective real divisor as the divisorial Zariski decomposition of $\alpha$, in terms of the non-Kähler locus of $p$ (cf. Section 3.5).

The geometric picture is now as follows: the pseudo-effective cone of a compact complex manifold $X$ is locally polyhedral away from the modified nef cone, with extremal rays that write $\mathbf{R}_{+}\{D\}$ for some exceptional prime $D$ of $X$. The Zariski projection $Z$ yields a projection from the pseudo-effective cone to the modified nef cone parallel to these exceptional rays, which map is concave (in some sense) and homogeneous, but not continuous up to the boundary of the pseudo-effective cone in general. The fibre $Z^{-1}(p)$ of $Z$ above a modified nef class $p$ is a countable union of simplicial cones generated by exceptional families of primes. 
When $X$ is a surface, a modified nef class is just a nef class; when $\alpha$ is the class of an effective Q-divisor $D$ on a projective surface, the divisorial Zariski decomposition of $\alpha$ is just the original Zariski decomposition of $D$. More generally, we show that the divisorial decomposition of a pseudo-effective class $\alpha$ on a Kähler surface is the unique orthogonal decomposition of $\alpha$ into the sum of a modified nef class and the class of an exceptional (in some sense) effective $\mathbf{R}$-divisor. This fact accounts for the rationality of the Zariski decomposition on a surface, meaning that the negative part $N(\alpha)$ is rational when $\alpha$ is.

An interesting fact is that much of the well-known case of a surface carries on to the case where $X$ is a compact hyper-Kähler manifold. Using the quadratic Beauville-Bogomolov form on $H^{1,1}(X, \mathbf{R})$ and deep results due to D. Huybrechts, we can prove the following facts: a family of primes is exceptional in our sense iff the corresponding Gram matrix is negative definite. In particular, a prime is exceptional iff it has negative square, and this forces it to be uniruled (Proposition 4.7). The modified nef cone of a hyper-Kähler manifold is just the dual cone to the pseudoeffective cone, which is also the closure of the so-called birational (or bimeromorphic) Kähler cone. Finally, the divisorial Zariski decomposition is the unique orthogonal decomposition into the sum of a modified nef class and an exceptional divisor. In particular, the divisorial Zariski decomposition is also rational in that case.

In a last part, we explain how to tackle the above constructions in a more algebraic fashion. When $L$ is a big $\mathbf{R}$-divisor on a projective manifold, we prove that the divisorial Zariski projection of $L$ is the only decomposition $L=P+N$ into real divisors with $P$ modified nef and $H^{0}(\lfloor k P\rfloor)=H^{0}(\lfloor k L\rfloor)$ for every $k$. The minimal multiplicities of $\{L\}$ (and thus its negative part) can be recovered from the asymptotic behaviour of the sections of $k L$. The case of a general pseudo-effective line bundle $L$ is then handled by approximating it by $L+\varepsilon A$, where $A$ is ample.

Shortly after this paper was completed, we have been informed by R. Lazarsfeld of an unpublished work of N. Nakayama [16] in which (among other things) the algebraic version of our constructions is studied in detail, in the case of a pseudo-effective $\mathbf{R}$-divisor on a projective manifold. Following the advice of the referee, we have included as an appendix a brief summary of some aspects of [16] which may prove interesting in the perspective of the present work.

\section{Technical preliminaries}

\section{1. $\partial \bar{\partial}-\operatorname{cohomology}$}

When $X$ is an arbitrary complex manifold, the $\partial \bar{\partial}$-lemma of Kähler geometry does not hold, and it is thus better to work with $\partial \bar{\partial}$-cohomology. We will just need the $(1,1)$-cohomology space $H_{\partial \bar{\partial}}^{1,1}(X, \mathbf{C})$, which is defined as the quotient of the space of $d$-closed smooth $(1,1)$-forms modulo the $\partial \bar{\partial}$-exact ones. The real structure on the space of forms induces a real structure on $H_{\partial \bar{\partial}}^{1,1}(X, \mathbf{C})$, and we denote by $H_{\partial \bar{\partial}}^{1,1}(X, \mathbf{R})$ the space of real points.

The canonical map from $H_{\partial \bar{\partial}}^{1,1}(X, \mathbf{C})$ to the quotient of the space of $d$-closed $(1,1)$-currents modulo the $\partial \bar{\partial}$-exact ones is injective (because, for any degree 0 current $f, \partial \bar{\partial} f$ is smooth iff $f$ is), and is also surjective: given a closed $(1,1)$-current $T$, one can find a locally finite open covering $U_{j}$ of $X$ such that $T=\partial \bar{\partial} f_{j}$ is $\partial \bar{\partial}$-exact on $U_{j}$. If $\rho_{j}$ is a partition of unity associated to $U_{j}$ and $f:=\sum \rho_{j} f_{j}$, then $T-\partial \bar{\partial} f$ is smooth. Indeed, on $U_{i}$, it is just $\partial \bar{\partial} \sum_{j} \rho_{j}\left(f_{i}-f_{j}\right)$, and each $f_{i}-f_{j}$ is smooth since it is even pluri-harmonic. As a consequence, a class $\alpha \in H_{\partial \bar{\partial}}^{1,1}(X, \mathbf{C})$ can be seen as an affine space of closed $(1,1)$-currents. We denote by $\{T\} \in H_{\partial \bar{\partial}}^{1,1}(X, \mathbf{C})$ the class of the current $T$. Remark that $i \partial \bar{\partial}$ is a real operator (on forms or currents), so that if $T$ 
is a real closed $(1,1)$-current, its class $\{T\}$ lies in $H_{\partial \bar{\partial}}^{1,1}(X, \mathbf{R})$ and consists of all the closed currents $T+i \partial \bar{\partial} \varphi$ where $\varphi$ is a real current of degree 0 .

When $X$ is furthermore compact, it can be shown that $H_{\partial \bar{\partial}}^{1,1}(X, \mathbf{C})$ is finite dimensional. The operator $\partial \bar{\partial}$ from smooth functions to smooth closed $(1,1)$-forms is thus an operator between Fréchet spaces with finite codimensional range; it therefore has closed range, and the quotient map $\theta \mapsto\{\theta\}$ from smooth closed $(1,1)$-forms to $H_{\partial \bar{\partial}}^{1,1}(X, \mathbf{C})$ endowed with its unique finitedimensional complex vector space Hausdorff topology is thus continuous and open.

\subsection{General facts about currents}

\subsubsection{Siu decomposition}

Let $T$ be a closed positive current of bidegree $(p, p)$ on a complex $n$-fold $X$. We denote by $\nu(T, x)$ its Lelong number at a point $x \in X$. The Lelong super-level sets are defined by $E_{c}(T):=\{x \in X, \nu(T, x) \geqslant c\}$ for $c>0$, and a well known result of Y.T. Siu [18] asserts that $E_{c}(T)$ is an analytic subset of $X$, of codimension at least $p$. As a consequence, for any analytic subset $Y$ of $X$, the generic Lelong number of $T$ along $Y$, defined by

$$
\nu(T, Y):=\inf \{\nu(T, x), x \in Y\},
$$

is also equal to $\nu(T, x)$ for a very general $x \in Y$. It is also true that, for any irreducible analytic subset $Y$ of codimension $p$ in $X$, the current $T-\nu(T, Y)[Y]$ is positive. The symbol $[Y]$ denotes the integration current on $Y$, which is defined by integrating test forms on the smooth locus of $Y$. Since $E_{+}(T):=\bigcup_{c>0} E_{c}(T)$ is a countable union of $p$-codimensional analytic subsets, it contains an at most countable family $Y_{k}$ of $p$-codimensional irreducible analytic subsets. By what we have said, $T-\nu\left(T, Y_{1}\right)\left[Y_{1}\right]-\cdots-\nu\left(T, Y_{k}\right)\left[Y_{k}\right]$ is a positive current for all $k$, thus the series $\sum_{k \geqslant 0} \nu\left(T, Y_{k}\right)\left[Y_{k}\right]$ converges, and we have

$$
T=R+\sum_{k} \nu\left(T, Y_{k}\right)\left[Y_{k}\right]
$$

for some closed positive $(p, p)$-current $R$ such that each $E_{c}(R)$ has codimension $>p$. The decomposition above is called the Siu decomposition of the closed positive $(p, p)$-current $T$. Since $\nu(T, Y)=0$ if $Y$ is a $p$-codimensional subvariety not contained in $E_{+}(T)$, it makes sense to write $\sum_{k} \nu\left(T, Y_{k}\right)\left[Y_{k}\right]=\sum \nu(T, Y)[Y]$, where the sum is implicitely extended over all $p$-codimensional irreducible analytic subsets $Y \subset X$.

\subsubsection{Almost positive currents}

A real $(1,1)$-current $T$ on a complex manifold $X$ is said to be almost positive if $T \geqslant \gamma$ holds for some smooth real $(1,1)$-form $\gamma$. Let $T \geqslant \gamma$ be a closed almost positive $(1,1)$-current. On a small enough open set $U$ with coordinates $z=\left(z_{1}, \ldots, z_{n}\right)$, we write $T=\partial \bar{\partial} \varphi$ where $\varphi$ is a degree 0 current. Since $\gamma+C i \partial \bar{\partial}|z|^{2}$ is a positive $(1,1)$-form on $U$ for $C>0$ big enough, we get that $i \partial \bar{\partial}\left(\varphi+C|z|^{2}\right)$ is positive, which means that $\varphi+C|z|^{2}$ is (the current associated to) a (unique) pluri-subharmonic function on $U$. A locally integrable function $\varphi$ on $X$ such that $i \partial \bar{\partial} \varphi$ is almost positive is called an almost pluri-subharmonic function, and is thus locally equal to a pluri-subharmonic function modulo a smooth function.

The Lelong number $\nu(T, x)$ of a closed almost positive $(1,1)$-current $T$ can be defined as $\nu\left(T+C i \partial \bar{\partial}|z|^{2}, x\right)$ as above, since this does not depend on the smooth function $C|z|^{2}$. Consequently, the Siu decomposition of $T$ can also be constructed, and writes

$$
T=R+\sum \nu(T, D)[D],
$$


where $D$ ranges over the prime divisors of $X$, and $R$ is a closed almost positive $(1,1)$-current. In fact, we have $R \geqslant \gamma$ as soon as $T \geqslant \gamma$ for a smooth form $\gamma$.

\subsubsection{Pull-back of a current}

When $f: Y \rightarrow X$ is a surjective holomorphic map between compact complex manifolds and $T$ is a closed almost positive $(1,1)$-current on $X$, it is possible to define its pull back $f^{\star} T$ by $f$ using the analogue of local equations for divisors: write $T=\theta+i \partial \bar{\partial} \varphi$ for some smooth form $\theta \in\{T\} . \varphi$ is then an almost pluri-subharmonic function, thus locally a plurisubharmonic function modulo $\mathcal{C}^{\infty}$. One defines $f^{\star} T$ to be $f^{\star} \theta+i \partial \bar{\partial} f^{\star} \varphi$, as this is easily seen to be independent of the choices made. Of course, we then have $\left\{f^{\star} T\right\}=f^{\star}\{T\}$.

\subsubsection{Gauduchon metrics and compactness}

On any compact complex $n$-fold $X$, there exists a Hermitian metric $\omega$ such that $\omega^{n-1}$ is $\partial \bar{\partial}$-closed. Such a metric is called a Gauduchon metric. As a consequence, for every smooth real $(1,1)$-form $\gamma$, the quotient map $T \mapsto\{T\}$ from the set of closed $(1,1)$-currents $T$ with $T \geqslant \gamma$ to $H_{\partial \bar{\partial}}^{1,1}(X, \mathbf{R})$ is proper. Indeed, the mass of the positive current $T-\gamma$ is controled by $\int(T-\gamma) \wedge \omega^{n-1}$, and $\int T \wedge \omega^{n-1}=\{T\} \cdot\{\omega\}$ only depends on the class of $T$. The result follows by the weak compactness of positive currents with bounded mass. Another consequence is that the kernel of $T \mapsto\{T\}$ meets the cone of closed positive $(1,1)$-currents at the origin only.

\subsection{Cones in the $\partial \bar{\partial}$-cohomology}

We now assume that $X$ is compact, and fix some reference Hermitian form $\omega$ (i.e. a smooth positive definite $(1,1)$-form). A cohomology class $\alpha \in H_{\partial \bar{\partial}}^{1,1}(X, \mathbf{R})$ is said to be pseudo-effective iff it contains a positive current; $\alpha$ is nef (numerically effective) iff, for each $\varepsilon>0, \alpha$ contains a smooth form $\theta_{\varepsilon}$ with $\theta_{\varepsilon} \geqslant-\varepsilon \omega ; \alpha$ is big iff it contains a Kähler current, i.e. a closed $(1,1)$-current $T$ such that $T \geqslant \varepsilon \omega$ for $\varepsilon>0$ small enough. Finally, $\alpha$ is a Kähler class iff it contains a Kähler form (note that a smooth Kähler current is the same thing as a Kähler form). Since any two Hermitian forms $\omega_{1}, \omega_{2}$ are commensurable (i.e. $C^{-1} \omega_{2} \leqslant \omega_{1} \leqslant C \omega_{2}$ for some $C>0$ ), these definitions do not depend on the choice of $\omega$.

The set of pseudo-effective classes is a closed convex cone $\mathcal{E} \subset H_{\partial \bar{\partial}}^{1,1}(X, \mathbf{R})$, called the pseudo-effective cone. It has compact base, because so is the case of the cone of closed positive $(1,1)$-currents. Similarly, one defines the nef cone $\mathcal{N}$ (a closed convex cone), the big cone $\mathcal{B}$ (an open convex cone), and the Kähler cone $\mathcal{K}$ (an open convex cone). We obviously have the inclusions

$$
\mathcal{K} \subset \mathcal{B} \subset \mathcal{E} \text { and } \mathcal{K} \subset \mathcal{N} \subset \mathcal{E} .
$$

By definition, $X$ is a Kähler manifold iff its Kähler cone $\mathcal{K}$ is non-empty. Similarly (but this is a theorem, cf. [7]) $X$ is a Fujiki manifold (i.e. bimeromorphic to a Kähler manifold) iff its big cone $\mathcal{B}$ is non-empty (see also the proof of Proposition 2.3 below). If $X$ is Kähler, $\mathcal{K}$ is trivially the interior of the nef cone. Similarly, if $X$ is Fujiki, $\mathcal{B}$ is trivially the interior of the pseudo-effective cone.

We will now and then denote by $\geqslant$ the partial order relation on $H_{\partial \bar{\partial}}^{1,1}(X, \mathbf{R})$ induced by the convex cone $\mathcal{E}$.

\subsection{The Néron-Severi space}

Given a line bundle $L$ on $X$, each smooth Hermitian metric $h$ on $L$ locally writes as $h(x, v)=|v|^{2} \mathrm{e}^{-2 \varphi(x)}$ for some smooth local weight $\varphi$; the curvature form $\Theta_{h}(L):=\frac{i}{\pi} \partial \bar{\partial} \varphi$ is a 
globally defined real $(1,1)$-form, whose class in $H_{\partial \bar{\partial}}^{1,1}(X, \mathbf{R})$ we denote by $c_{1}(L)$, the first Chern class of $L$. We write $d d^{c}=\frac{i}{\pi} \partial \bar{\partial}$ for short. A singular Hermitian metric $h$ on $L$ is by definition a metric $h=h_{\infty} \mathrm{e}^{-2 \varphi}$, where $h_{\infty}$ is a smooth Hermitian metric on $L$ and the weight $\varphi$ is a locally integrable function. The curvature current of $h$ is defined as $\Theta_{h}(L):=\Theta_{h_{\infty}}(L)+d d^{c} \varphi$; it also lies in $c_{1}(L)$. Conversely, given a smooth Hermitian metric $h_{\infty}$ on $L$, any closed real $(1,1)$-current $T$ in $c_{1}(L)$ can be written (by definition) as $T=\Theta_{h_{\infty}}(L)+d d^{c} \varphi$. But $\varphi$ is just a degree 0 current a priori. However, $\varphi$ is automatically $L_{\text {loc }}^{1}$ in case $T$ is almost positive (cf. Section 2.2.2), thus each almost positive current $T$ in $c_{1}(L)$ is the curvature form of a singular Hermitian metric on $L$.

The image of the homomorphism $\operatorname{Pic}(X) \rightarrow H_{\partial \bar{\partial}}^{1,1}(X, \mathbf{R}) L \mapsto c_{1}(L)$ is called the NéronSeveri group, denoted by $N S(X)$. Its rank is denoted by $\rho(X)$, and called the Picard number of $X$. The real Néron-Severi space $N S(X)_{\mathbf{R}}$ is just the real subspace of dimension $\rho(X)$ in $H_{\partial \bar{\partial}}^{1,1}(X, \mathbf{R})$ generated by $N S(X)$. Kodaira's embedding theorem can be formulated as follows: $X$ is a projective manifold iff the intersection of the Kähler cone $\mathcal{K}$ with $N S(X)_{\mathbf{R}}$ is nonempty. Similarly, $X$ is a Moishezon manifold (i.e. bimeromorphic to a projective manifold) iff the intersection of the big cone $\mathcal{B}$ with $N S(X)_{\mathbf{R}}$ is non-empty (cf. [7]).

\subsection{Currents with analytic singularities}

\subsubsection{Definition}

A closed almost positive $(1,1)$-current $T$ on a compact complex $n$-fold $X$ is said to have analytic singularities (along a subscheme $V(\mathcal{I})$ defined by a coherent ideal sheaf $\mathcal{I}$ ) if there exists some $c>0$ such that $T$ is locally congruent to $\frac{c}{2} d d^{c} \log \left(\left|f_{1}\right|^{2}+\cdots+\left|f_{k}\right|^{2}\right)$ modulo smooth forms, where $f_{1}, \ldots, f_{k}$ are local generators of $\mathcal{I}$. $T$ is thus smooth outside the support of $V(\mathcal{I})$, and it is an immediate consequence of the Lelong-Poincaré formula that $\sum \nu(T, D) D$ is just $c$ times the divisor part of the scheme $V(\mathcal{I})$. If we first blow-up $X$ along $V(\mathcal{I})$ and then resolve the singularities, we get a modification $\mu: \widetilde{X} \rightarrow X$, where $\widetilde{X}$ is a compact complex manifold, such that $\mu^{-1} \mathcal{I}$ is just $\mathcal{O}(-D)$ for some effective divisor $D$ upstairs. The pull-back $\mu^{\star} T$ clearly has analytic singularities along $V\left(\mu^{-1} \mathcal{I}\right)=D$, thus its Siu decomposition writes

$$
\mu^{\star} T=\theta+c D
$$

where $\theta$ is a smooth $(1,1)$-form. If $T \geqslant \gamma$ for some smooth form $\gamma$, then $\mu^{\star} T \geqslant \mu^{\star} \gamma$, and thus $\theta \geqslant \mu^{\star} \gamma$. This operation we call a resolution of the singularities of $T$.

\subsubsection{Regularization(s) of currents}

We will need two basic types of regularizations (inside a fixed cohomology class) for closed $(1,1)$-currents, both due to J.-P. Demailly.

THEOREM $2.1[4,5]$. - Let $T$ be a closed almost positive $(1,1)$-current on a compact complex manifold $X$, and fix a Hermitian form $\omega$. Suppose that $T \geqslant \gamma$ for some smooth real $(1,1)$-form $\gamma$ on $X$. Then:

(i) There exists a sequence of smooth forms $\theta_{k}$ in $\{T\}$ which converges weakly to $T$, and such that $\theta_{k} \geqslant \gamma-C \lambda_{k} \omega$ where $C>0$ is a constant depending on the curvature of $\left(T_{X}, \omega\right)$ only, and $\lambda_{k}$ is a decreasing sequence of continuous functions such that $\lambda_{k}(x) \rightarrow \nu(T, x)$ for every $x \in X$.

(ii) There exists a sequence $T_{k}$ of currents with analytic singularities in $\{T\}$ which converges weakly to $T$, such that $T_{k} \geqslant \gamma-\varepsilon_{k} \omega$ for some sequence $\varepsilon_{k}>0$ decreasing to 0 , and such that $\nu\left(T_{k}, x\right)$ increases to $\nu(T, x)$ uniformly with respect to $x \in X$. 
Point (ii) enables us in particular to approximate a Kähler current $T$ inside its cohomology class by Kähler currents $T_{k}$ with analytic singularities, with a very good control of the singularities. A big class therefore contains plenty of Kähler currents with analytic singularities.

\subsection{Intersection of currents}

Just as cycles, currents can be intersected provided their singular sets are in an acceptable mutual position. Specifically, let $T$ be a closed positive $(1,1)$-current on a complex manifold $X$. Locally, we have $T=d d^{c} \varphi$ with $\varphi$ a pluri-subharmonic function, which is well defined modulo a pluri-harmonic (hence smooth) function. We therefore get a globally well-defined unbounded locus $L(T)$, which is the complement of the open set of points near which $\varphi$ is locally bounded. Assume now that $T_{1}, T_{2}$ are two closed positive $(1,1)$-currents such that $L\left(T_{j}\right)$ is contained in an analytic set $A_{j}$ (which may be $X$ ); locally, we write $T_{j}=d d^{c} \varphi_{j}$ with $\varphi_{j}$ a pluri-subharmonic function. If $A_{1} \cap A_{2}$ has codimension at least 2 , then it is shown in [5] that $\varphi_{1} d d^{c} \varphi_{2}$ has locally finite mass, and that $d d^{c} \varphi_{1} \wedge d d^{c} \varphi_{2}:=d d^{c}\left(\varphi_{1} d d^{c} \varphi_{2}\right)$ yields a globally defined closed positive $(2,2)$-current, denoted by $T_{1} \wedge T_{2}$. It is also true that $T_{1} \wedge T_{2}$ lies in the product cohomology class $\left\{T_{1}\right\} \cdot\left\{T_{2}\right\} \in H_{\partial \bar{\partial}}^{2,2}(X, \mathbf{R})$.

We will only need the following two special cases: if $T_{1}$ is a closed positive $(1,1)$-current with analytic singularities along a subscheme of codimension at least 2 , then $T_{1} \wedge T_{2}$ exists for every closed positive $(1,1)$-current $T_{2}$.

If $D_{1}$ and $D_{2}$ are two distinct prime divisors, then $\left[D_{1}\right] \wedge\left[D_{2}\right]$ is a well defined closed positive $(2,2)$-current. Since its support is clearly contained in the set-theoretic intersection $D_{1} \cap D_{2}$ (whose codimension is at least 2), we have $\left[D_{1}\right] \wedge\left[D_{2}\right]=\sum a_{j}\left[Y_{j}\right]$, where the $Y_{j}$ 's are the components of $D_{1} \cap D_{2}$. In fact, it can be shown that $\sum a_{j} Y_{j}$ is just the 2 -cycle associated to the scheme-theoretic intersection $D_{1} \cap D_{2}$, thus $\left[D_{1}\right] \wedge\left[D_{2}\right]$ is just the integration current associated to the cycle $D_{1} \cdot D_{2}$.

\subsection{The modified nef cone}

For our purposes, we need to introduce a new cone in $H_{\partial \bar{\partial}}^{1,1}(X, \mathbf{R})$, which is somehow the cone of classes that are nef in codimension 1 . Let $X$ be a compact complex $n$-fold, and $\omega$ be some reference Hermitian form.

Definition 2.2 (Modified nef and Kähler classes). - Let $\alpha$ be a class in $H_{\partial \bar{\partial}}^{1,1}(X, \mathbf{R})$.

(i) $\alpha$ is said to be a modified Kähler class iff it contains a Kähler current $T$ with $\nu(T, D)=0$ for all prime divisors $D$ in $X$.

(ii) $\alpha$ is said to be a modified nef class iff, for every $\varepsilon>0$, there exists a closed $(1,1)$-current $T_{\varepsilon}$ in $\alpha$ with $T_{\varepsilon} \geqslant-\varepsilon \omega$ and $\nu\left(T_{\varepsilon}, D\right)=0$ for every prime $D$.

This is again independent of the choice of $\omega$ by commensurability of the Hermitian forms. The set of modified Kähler classes is an open convex cone called the modified Kähler cone and denoted by $\mathcal{M K}$. Similarly, we get a closed convex cone $\mathcal{M N}$, the modified nef cone. Using the Siu decomposition, we immediately see that $\mathcal{M K}$ is non-empty iff the big cone $\mathcal{B}$ is non-empty, in which case $\mathcal{M K}$ is just the interior of $\mathcal{M N}$.

Remark 1. - Upon regularizing the currents using (ii) of Theorem 2.1, we can always assume that the currents involved in the definition have analytic singularities along a subscheme of codimension at least 2 .

Remark 2. - The modified nef cone of a compact complex surface is just its nef cone (cf. Section 4.2.1). 
Remark 3.- Just as for nef classes, one cannot simply take $\varepsilon=0$ in the definition of a modified nef class. We recall the example given in [8]: there exists a ruled surface $X$ over an elliptic curve such that $X$ contains an irreducible curve $C$ with the following property: the class $\{C\} \in H_{\partial \bar{\partial}}^{1,1}(X, \mathbf{R})$ is nef, but contains only one positive current, which is of course the integration current $[C]$.

The following proposition gives a more "algebraic" characterization of $\mathcal{M K}$, which also explains the (seemingly dumb) terminology.

Proposition 2.3. - A class $\alpha$ lies in $\mathcal{M K}$ iff there exist a modification $\mu: \widetilde{X} \rightarrow X$ and a Kähler class $\widetilde{\alpha}$ on $\widetilde{X}$ such that $\alpha=\mu_{\star} \widetilde{\alpha}$.

Proof. - The argument is adapted from [7], Theorem 3.4. If $\widetilde{\omega}$ is a Kähler form on $\widetilde{X}$ and $\omega$ is our reference Hermitian form on $X$, then $\mu^{\star} \omega \leqslant C \widetilde{\omega}$ for some $C>0$, since $\widetilde{X}$ is compact. Since $\mu$ is a modification, we have $\mu_{\star} \mu^{\star} \omega=\omega$, so we get $T:=\mu_{\star} \widetilde{\omega} \geqslant C^{-1} \omega$, and $T$ is thus a Kähler current. Since the singular values of $\mu$ are in codimension at least 2 , we immediately see that $\nu(T, D)=0$ for every prime divisor $D$ in $X$, and $\{T\}=\mu_{\star}\{\omega\}$ lies in $\mathcal{M K}$ as desired. Conversely, if $\alpha \in \mathcal{M K}$ is represented by a Kähler current $T$ with $\nu(T, D)=0$ for all $D$, there exists by (ii) of Theorem 2.1 a Kähler current $T_{k}$ in $\alpha$ with analytic singularities along a subscheme $V_{k}$ with $\nu\left(T_{k}, D\right) \leqslant \nu(T, D)$, so that $V_{k}$ has no divisor component. We select a resolution of the singularities of $T_{k} \mu: \widetilde{X} \rightarrow X$, and write $\mu^{\star} T_{k}=\theta+F$, where $\theta$ is a smooth form and $F$ is an effective $\mathbf{R}$-divisor. Since $T_{k} \geqslant \varepsilon \omega$ for $\varepsilon>0$ small enough, we get that $\theta \geqslant \mu^{\star} \varepsilon \omega$. Denoting by $E_{1}, \ldots, E_{r}$ the $\mu$-exceptional prime divisors on $\widetilde{X}$, it is shown in [7], Lemma 3.5, that one can find $\delta_{1}, \ldots, \delta_{r}>0$ small enough and a closed smooth $(1,1)$-form $\tau$ in $\left\{\delta_{1} E_{1}+\cdots+\delta_{r} E_{r}\right\}$ such that $\mu^{\star} \varepsilon \omega-\tau$ is positive definite everywhere. It follows that $\theta-\tau$ is a Kähler form upstairs. Now, we have

$$
\alpha=\mu_{\star}\left\{T_{k}\right\}=\mu_{\star}\left\{\theta-\left(\delta_{1} E_{1}+\cdots+\delta_{r} E_{r}\right)\right\}=\mu_{\star}\{\theta-\tau\},
$$

since $E_{j}$ is $\mu$-exceptional and so is $F$ because $\mu_{\star} F$ is an effective divisor contained in the scheme $V_{k}$; this concludes the proof of Proposition 2.3.

That a modified nef class is somehow nef in codimension 1 is reflected in the following

PROPOSITION 2.4. - If $\alpha$ is a modified Kähler (respectively nef) class, then $\alpha_{\mid D}$ is big (respectively pseudo-effective) for every prime divisor $D \subset X$.

Proof. - If $\alpha$ is a modified nef class and $\varepsilon>0$ is given, choose a current $T_{\varepsilon} \geqslant-\varepsilon \omega$ in $\alpha$ with analytic singularities in codimension at least 2. Locally, we have $\omega \leqslant C d d^{c}|z|^{2}$ for some $C>0$, thus $T_{\varepsilon}+\varepsilon C d d^{c}|z|^{2}$ writes as $d d^{c} \varphi_{\varepsilon}$, where $\varphi_{\varepsilon}$ is pluri-subharmonic and is not identically $-\infty$ on $D$. Thus the restriction $\left(\varphi_{\varepsilon}\right)_{\mid D}$ is pluri-subharmonic, and $\left(T_{\varepsilon}+\varepsilon C d d^{c}|z|^{2}\right)_{\mid D}$ is a well defined closed positive current. It follows that $\left(T_{\varepsilon}\right)_{\mid D}$ is a well defined almost positive current on $D$, with $\left(T_{\varepsilon}\right)_{\mid D} \geqslant-\varepsilon C \omega_{\mid D}$. This certainly implies that $\alpha_{\mid D}$ is pseudo-effective. The case $\alpha \in \mathcal{M K}$ is treated similarly.

\subsection{Currents with minimal singularities}

Let $\varphi_{1}, \varphi_{2}$ be two almost pluri-subharmonic functions on a compact complex manifold $X$. Then, following [10], we say that $\varphi_{1}$ is less singular than $\varphi_{2}$ (and write $\varphi_{1} \preceq \varphi_{2}$ ) if we have $\varphi_{2} \leqslant \varphi_{1}+C$ for some constant $C$. We denote by $\varphi_{1} \approx \varphi_{2}$ the equivalence relation generated by the pre-order relation $\preceq$. Note that $\varphi_{1} \approx \varphi_{2}$ exactly means that $\varphi_{1}=\varphi_{2} \bmod L^{\infty}$. 
When $T_{1}$ and $T_{2}$ are two closed almost positive $(1,1)$-currents on $X$, we can also compare their singularities in the following fashion: write $T_{i}=\theta_{i}+d d^{c} \varphi_{i}$ for $\theta_{i} \in\left\{T_{j}\right\}$ a smooth form and $\varphi_{i}$ an almost pluri-subharmonic function. Since any $L_{\text {loc }}^{1}$ function $f$ with $d d^{c} f$ smooth is itself smooth, it is easy to check that $\varphi_{i}$ does not depend on the choices made up to equivalence of singularities, and we compare the singularities of the $T_{i}$ 's by comparing those of the $\varphi_{i}$ 's.

Let now $\alpha$ be a class in $H_{\partial \bar{\partial}}^{1,1}(X, \mathbf{R})$ and $\gamma$ be a smooth real $(1,1)$-form, and denote by $\alpha[\gamma]$ the set of closed almost positive $(1,1)$-currents $T$ lying in $\alpha$ with $T \geqslant \gamma$. It is a (weakly) compact and convex subset of the space of $(1,1)$-currents. We endow it with the pre-order relation $\preceq$ defined above. For any family $T_{j}, j \in J$ of elements of $\alpha[\gamma]$, we claim that there exists an infimum $T=\inf _{j \in J} T_{j}$ in $(\alpha[\gamma], \preceq)$, which is therefore unique up to equivalence of singularities. The proof is pretty straightforward: fix a smooth form $\theta$ in $\alpha$, and write $T_{j}=\theta+d d^{c} \varphi_{j}$ for some quasi pluri-subharmonic functions $\varphi_{j}$. Since $X$ is compact, $\varphi_{j}$ is bounded from above; therefore, upon changing $\varphi_{j}$ into $\varphi_{j}-C_{j}$, we may assume that $\varphi_{j} \leqslant 0$ for all $j \in J$. We then take $\varphi$ to be the upper semi-continuous upper envelope of the $\varphi_{j}$ 's, $j \in J$, and set $T:=\theta+d d^{c} \varphi$. It is immediate to check that $T \preceq T_{j}$ for all $j$, and that for every $S \in \alpha[\gamma], S \preceq T_{j}$ for all $j$ implies that $S \preceq T$. We should maybe explain why $T \geqslant \gamma$ : locally, we can choose coordinates $z=\left(z_{1}, \ldots, z_{n}\right)$ and a form $q(z)=\sum \lambda_{j}\left|z_{j}\right|^{2}$ such that $d d^{c} q \leqslant \gamma$ and $d d^{c} q$ is arbitrarily close to $\gamma$. Writing $\theta=d d^{c} \psi$ for some smooth local potential $\psi$, the condition $\theta+d d^{c} \varphi_{j} \geqslant \gamma$ implies that $\psi+\varphi_{j}-q$ is plurisubharmonic. The upper envelope $\psi+\varphi-q$ is thus also pluri-subharmonic, which means that $T=\theta+d d^{c} \varphi \geqslant d d^{c} q$; letting $d d^{c} q$ tend to $\gamma$, we get $T \geqslant \gamma$, as desired.

Since any two closed almost positive currents with equivalent singularities have the same Lelong numbers, the Lelong numbers of $\inf T_{j}$ do not depend on the specific choice of the current. In fact, it is immediate to check from the definitions that

$$
\nu\left(\inf _{j \in J} T_{j}, x\right)=\inf _{j \in J} \nu\left(T_{j}, x\right) .
$$

As a particular case of the above construction, there exists a closed almost positive $(1,1)$-current $T_{\min , \gamma} \in \alpha[\gamma]$ which is a least element in $(\alpha[\gamma], \preceq) . T_{\min , \gamma}$ is well defined modulo $d d^{c} L^{\infty}$, and we call it a current with minimal singularities in $\alpha$, for the given lower bound $\gamma$. When $\gamma=0$ and $\alpha$ is pseudo-effective, we just write $T_{\min }=T_{\min , 0}$, and call it a positive current with minimal singularities in $\alpha$. It must be noticed that, even for a big class $\alpha, T_{\min }$ will be a Kähler current only in the trivial case:

Proposition 2.5. - A pseudo-effective class $\alpha$ contains a positive current with minimal singularities $T_{\min }$ which is a Kähler current iff $\alpha$ is a Kähler class.

Proof. - We can write $T_{\min }=\theta+d d^{c} \varphi$ with $\theta$ a smooth form. If $T_{\min }$ is Kähler, then so is $\varepsilon \theta+(1-\varepsilon) T_{\min }=\theta+d d^{c}(1-\varepsilon) \varphi$ for $\varepsilon>0$ small enough. We therefore get $\varphi \preceq(1-\varepsilon) \varphi$ by minimality, that is: $(1-\varepsilon) \varphi \leqslant \varphi+C$ for some constant $C$. But this shows that $\varphi$ is bounded, and thus $T_{\min }$ is a Kähler current with identically zero Lelong numbers. Using (i) of Theorem 2.1, we can therefore regularize it into a Kähler form inside its cohomology class.

Finally, we remark that a positive current with minimal singularities in a pseudo-effective class is generally non-unique (as a current), as the example of a Kähler class already shows.

\section{The divisorial Zariski decomposition}

In this section $X$ denotes a compact complex $n$-fold, and $\omega$ is a reference Hermitian form, unless otherwise specified. 


\subsection{Minimal multiplicities and non-nef locus}

When $\alpha \in H_{\partial \bar{\partial}}^{1,1}(X, \mathbf{R})$ is a pseudo-effective class, we want to introduce minimal multiplicities $\nu(\alpha, x)$, which measure the obstruction to the numerical effectivity of $\alpha$. For each $\varepsilon>0$, let $T_{\min , \varepsilon}=T_{\min , \varepsilon}(\alpha)$ be a current with minimal singularities in $\alpha[-\varepsilon \omega]$ (cf. Section 2.8 for the notation). We then introduce the following

DEFINITION 3.1 (Minimal multiplicities). - The minimal multiplicity at $x \in X$ of the pseudoeffective class $\alpha \in H_{\partial \bar{\partial}}^{1,1}(X, \mathbf{R})$ is defined as

$$
\nu(\alpha, x):=\sup _{\varepsilon>0} \nu\left(T_{\min , \varepsilon}, x\right) .
$$

The commensurability of any two Hermitian forms shows that the definition does not depend on $\omega$. Furthermore, we do have $\nu(\alpha, x)<+\infty$, since trivially $\nu(\alpha, x) \leqslant \nu(T, x)$ if $T$ is any closed positive current in $\alpha$. When $D$ is a prime divisor, we define the generic minimal multiplicity of $\alpha$ along $D$ as

$$
\nu(\alpha, D):=\inf \{\nu(\alpha, x), x \in D\} .
$$

We then have $\nu(\alpha, D)=\sup _{\varepsilon>0} \nu\left(T_{\min , \varepsilon}, D\right)$, and $\nu(\alpha, D)=\nu(\alpha, x)$ for the very general $x \in D$.

Proposition 3.2. - Let $\alpha \in H_{\partial \bar{\partial}}^{1,1}(X, \mathbf{R})$ be a pseudo-effective class.

(i) $\alpha$ is nef iff $\nu(\alpha, x)=0$ for every $x \in X$.

(ii) $\alpha$ is modified nef iff $\nu(\alpha, D)=0$ for every prime $D$.

Proof. - If $\alpha$ is nef (respectively modified nef), $\alpha[-\varepsilon \omega]$ contains by definition a smooth form (respectively a current $T_{\varepsilon}$ with $\nu\left(T_{\varepsilon}, D\right)=0$ for every prime $D$ ). We thus have $\nu\left(T_{\min , \varepsilon}, x\right)=0$ (respectively $\nu\left(T_{\min , \varepsilon}, D\right)=0$ ) for every $\varepsilon>0$, and thus $\nu(\alpha, x)=0$ (respectively $\nu(\alpha, D)=0$ ). Conversely, if $\nu(\alpha, x)=0$ for every $x \in X$, applying (i) of Theorem 2.1 to $T_{\min , \varepsilon}$, we see that $\nu\left(T_{\min , \varepsilon}, x\right)=0$ for every $x \in X$ implies that $\alpha\left[-\varepsilon^{\prime} \omega\right]$ contains a smooth form for every $\varepsilon^{\prime}>\varepsilon$, and $\alpha$ is thus nef. Finally, if $\nu(\alpha, D)=0$ for every prime $D$, we have $\nu\left(T_{\min , \varepsilon}, D\right)=0$ for every prime $D$. Since $T_{\min , \varepsilon}$ lies in $\alpha[-\varepsilon \omega], \alpha$ is modified nef by the very definition.

In view of Proposition 3.2, we propose the

DEFINITION 3.3 (Non-nef locus). - The non-nef locus of a pseudo-effective class $\alpha \in H_{\partial \bar{\partial}}^{1,1}(X, \mathbf{R})$ is defined by

$$
E_{n n}(\alpha):=\{x \in X, \nu(\alpha, x)>0\} .
$$

Recall that the set $E_{+}(T):=\{x \in X, \nu(T, x)>0\}$ is a countable union of closed analytic subsets for every closed almost positive $(1,1)$-current $T$. Since $E_{n n}(\alpha)=\bigcup_{\varepsilon>0} E_{+}\left(T_{\min , \varepsilon}\right)$, the non-nef locus is also a countable union of closed analytic subsets. We do not claim however that each super-level set $\{x \in X, \nu(\alpha, x) \geqslant c\}(c>0)$ is an analytic subset (this is most certainly not true in general). Using results of M. Paun, Proposition 3.2 generalizes as follows:

Proposition 3.4. - A pseudo-effective class $\alpha$ is nef iff $\alpha_{\mid Y}$ is pseudo-effective for every irreducible analytic subset $Y \subset E_{n n}(\alpha)$.

Proof. - Paun's result [17] states that a pseudo-effective class is nef iff its restriction to every irreducible analytic subset is nef. It is thus enough to notice the following fact: if $Y \subset X$ is an 
analytic subset not entirely contained in the non-nef locus of $\alpha$, then $\alpha_{\mid Y}$ is pseudo-effective. This is proved exactly as Proposition 2.4, replacing $D$ by $Y$ there.

We now investigate the continuity of $\alpha \mapsto \nu(\alpha, x)$ and $\nu(\alpha, D)$ :

Proposition 3.5. - For every $x \in X$ and every prime $D$, the maps $\mathcal{E} \rightarrow \mathbf{R} \alpha \mapsto \nu(\alpha, x)$ and $\nu(\alpha, D)$ are convex, homogeneous. They are continuous on the interior $\mathcal{E}^{0}$, and lower semicontinuous on the whole of $\mathcal{E}$.

Proof. - Let $\alpha, \beta$ be two pseudo-effective classes. If $T_{\min , \varepsilon}(\alpha)$ and $T_{\min , \varepsilon}(\beta)$ are currents with minimal singularities in $\alpha[-\varepsilon \omega]$ and $\beta[-\varepsilon \omega]$ respectively, then $T_{\min , \varepsilon}(\alpha)+T_{\min , \varepsilon}(\alpha)$ belongs to $(\alpha+\beta)[-2 \varepsilon \omega]$, thus

$$
\nu\left(T_{\min , 2 \varepsilon}(\alpha+\beta), x\right) \leqslant \nu\left(T_{\min , \varepsilon}(\alpha), x\right)+\nu\left(T_{\min , \varepsilon}(\beta), x\right) \leqslant \nu(\alpha, x)+\nu(\beta, x) .
$$

We infer from this $\nu(\alpha+\beta, x) \leqslant \nu(\alpha, x)+\nu(\beta, x)$, and a similar sub-additivity property for $\nu(\cdot, D)$ is obtained along the same lines. Since the homogeneity of our two maps is obvious, the convexity also follows.

The quotient map $\theta \mapsto\{\theta\}$ from the Fréchet space of closed smooth real (1,1)-forms to $H_{\partial \bar{\partial}}^{1,1}(X, \mathbf{R})$ is surjective, thus open. If $\alpha_{k} \in H_{\partial \bar{\partial}}^{1,1}(X, \mathbf{R})$ is a sequence of pseudo-effective classes converging to $\alpha$ and $\varepsilon>0$ is given, we can thus find a smooth form $\theta_{k} \in \alpha-\alpha_{k}$ for each $k$ big enough such that $-\varepsilon \omega \leqslant \theta_{k} \leqslant \varepsilon \omega$. The current $T_{\min , \varepsilon}\left(\alpha_{k}\right)+\theta_{k}$ then lies in $\alpha[-2 \varepsilon \omega]$, and thus $\nu\left(T_{\min , 2 \varepsilon}(\alpha), x\right) \leqslant \nu\left(T_{\min , \varepsilon}\left(\alpha_{k}\right), x\right) \leqslant \nu\left(\alpha_{k}, x\right)$, for each $k$ big enough. We infer from this that $\nu\left(T_{\min , 2 \varepsilon}(\alpha), x\right) \leqslant \liminf _{k \rightarrow \infty} \nu\left(\alpha_{k}, x\right)$ for each $\varepsilon>0$, hence

$$
\nu(\alpha, x) \leqslant \liminf _{k \rightarrow \infty} \nu\left(\alpha_{k}, x\right)
$$

by taking the supremum of the left hand side for $\varepsilon>0$. This means that $\alpha \mapsto \nu(\alpha, x)$ is lower semi-continuous, and similarly for $\nu(\alpha, D)$, just replacing $x$ by $D$ in the above proof.

Finally, the restrictions of our maps to $\mathcal{E}^{0}$ are continuous as any convex map on an open convex subset of a finite dimensional vector space is.

PROPOSITION 3.6. - Let $\alpha \in H_{\partial \bar{\partial}}^{1,1}(X, \mathbf{R})$ be a pseudo-effective class, and $T_{\min }$ be a positive current with minimal singularities in $\alpha$.

(i) We always have $\nu(\alpha, x) \leqslant \nu\left(T_{\min }, x\right)$ and $\nu(\alpha, D) \leqslant \nu\left(T_{\min }, D\right)$.

(ii) When $\alpha$ is furthermore big, we have $\nu(\alpha, x)=\nu\left(T_{\min }, x\right)$ and $\nu(\alpha, D)=\nu\left(T_{\min }, D\right)$.

Proof. - Since $T_{\min }$ belongs to $\alpha[-\varepsilon \omega]$ for every $\varepsilon>0, \nu(\alpha, x) \leqslant \nu\left(T_{\min }, x\right)$ follows for every $x \in X$, for any pseudo-effective class $\alpha$. If $\alpha$ is furthermore big, we can choose a Kähler current $T$ in $\alpha$ with $T \geqslant \omega$ for some Hermitian form $\omega$. If $T_{\min , \varepsilon}$ is a current with minimal singularities in $\alpha[-\varepsilon \omega]$, then $(1-\varepsilon) T_{\min , \varepsilon}+\varepsilon T$ is a positive current in $\alpha$, and thus $\nu\left((1-\varepsilon) T_{\min , \varepsilon}+\varepsilon T, x\right) \geqslant \nu\left(T_{\min }, x\right)$ by minimality of $T_{\min }$, from which we infer

$$
(1-\varepsilon) \nu(\alpha, x)+\varepsilon \nu(T, x) \geqslant \nu\left(T_{\min }, x\right) .
$$

We thus get the converse inequality $\nu(\alpha, x) \geqslant \nu\left(T_{\min }, x\right)$ by letting $\varepsilon \rightarrow 0$. The case of $\nu(\alpha, D)$ is similar.

\subsection{Definition of the divisorial Zariski decomposition}

Let $\alpha \in H_{\partial \bar{\partial}}^{1,1}(X, \mathbf{R})$ be again a pseudo-effective class, and choose a positive current with minimal singularities $T_{\min }$ in $\alpha$. Since $\nu(\alpha, D) \leqslant \nu\left(T_{\min }, D\right)$ for every prime $D$ by 
Proposition 3.8, the series of currents $\sum \nu(\alpha, D)[D]$ is convergent, since it is dominated by $\sum \nu\left(T_{\min }, D\right)[D]$.

DEFINITION 3.7 (Divisorial Zariski decomposition). - The negative part of a pseudo-effective class $\alpha \in H_{\partial \bar{\partial}}^{1,1}(X, \mathbf{R})$ is defined as $N(\alpha):=\sum \nu(\alpha, D)[D]$. The Zariski projection of $\alpha$ is $Z(\alpha):=\alpha-\{N(\alpha)\}$. We call the decomposition $\alpha=Z(\alpha)+\{N(\alpha)\}$ the divisorial Zariski decomposition of $\alpha$.

It is certainly highly desirable that the negative part $N(\alpha)$ of a pseudo-effective class be a divisor, i.e. that $\nu(\alpha, D)=0$ for almost every prime $D$. We will see in Section 3.3 that it is indeed the case. For the time being, we concentrate on the Zariski projection, which we see as a $\operatorname{map} Z: \mathcal{E} \rightarrow \mathcal{E}$.

PROPOSITION 3.8. - Let $\alpha \in H_{\partial \bar{\partial}}^{1,1}(X, \mathbf{R})$ be a pseudo-effective class. Then:

(i) Its Zariski projection $Z(\alpha)$ is a modified nef class.

(ii) We have $Z(\alpha)=\alpha$ iff $\alpha$ is modified nef.

(iii) $Z(\alpha)$ is big iff $\alpha$ is.

(iv) If $\alpha$ is not modified nef, then $Z(\alpha)$ belongs to the boundary $\partial \mathcal{M N}$ of the modified nef cone.

Proof. - (i) Let $T_{\min , \varepsilon}$ be as before a current with minimal singularities in $\alpha[-\varepsilon \omega]$, and consider its Siu decomposition $T_{\min , \varepsilon}=R_{\varepsilon}+\sum \nu\left(T_{\min , \varepsilon}, D\right)[D]$. First, we claim that $N_{\varepsilon}:=\sum \nu\left(T_{\min , \varepsilon}, D\right)[D]$ converges weakly to $N(\alpha)$ as $\varepsilon$ goes to 0 . For any smooth form $\theta$ of bidimension (1,1), $\theta+C \omega^{n-1}$ is a positive form for $C>0$ big enough. Every such $\theta$ is thus the difference of two positive forms, and it is enough to show that $\int N_{\varepsilon} \wedge \theta \rightarrow \int N(\alpha) \wedge \theta$ for every smooth positive form $\theta$. But $\int N_{\varepsilon} \wedge \theta=\sum \nu\left(T_{\min , \varepsilon}, D\right) \int[D] \wedge \theta$ is a convergent series whose general term $\nu\left(T_{\min , \varepsilon}, D\right) \int[D] \wedge \theta$ converges to $\nu(\alpha, D) \int[D] \wedge \theta$ as $\varepsilon \rightarrow 0$ and is dominated by $\nu\left(T_{\text {min }}, D\right) \int[D] \wedge \theta$; since $\sum \nu\left(T_{\min }, D\right) \int[D] \wedge \theta \leqslant \int T_{\min } \wedge \theta$ converges, our claim follows by dominated convergence.

In particular, the class $\left\{N_{\varepsilon}-N(\alpha)\right\}$ converges to zero. Since the map $\theta \mapsto\{\theta\}$ is open on the space of smooth closed $(1,1)$-form, we can find a sequence $\theta_{k} \geqslant-\delta_{k} \omega$ of smooth forms with $\theta_{k} \in\left\{N_{\varepsilon_{k}}-N(\alpha)\right\}$ for some sequences $\varepsilon_{k} \ll \delta_{k}$ going to zero. It remains to notice that $T_{k}:=R_{\varepsilon_{k}}+\theta_{k}$ is a current in $Z(\alpha)$ with $T_{k} \geqslant-\left(\varepsilon_{k}+\delta_{k}\right) \omega$ and $\nu\left(T_{k}, D\right)=0$ for every prime $D$. Since $\varepsilon_{k}+\delta_{k}$ converges to zero, $Z(\alpha)$ is modified nef by definition.

(ii) Since $N(\alpha)=\sum \nu(\alpha, D)[D]$ is a closed positive $(1,1)$-current, it is zero iff its class $\{N(\alpha)\} \in H_{\partial \bar{\partial}}^{1,1}(X, \mathbf{R})$ is. The assertion is thus just a reformulation of (ii) in Proposition 3.2.

(iii) If $Z(\alpha)$ is big, then of course $\alpha=Z(\alpha)+\{N(\alpha)\}$ is also big, as the sum of a big class and a pseudo-effective one. If conversely $\alpha$ is big, it contains a Kähler current $T$, whose Siu decomposition we write $T=R+\sum \nu(T, D)[D]$. Note that $R$ is a Kähler current since $T$ is; since $T$ belongs to $\alpha[-\varepsilon \omega]$ for every $\varepsilon>0$, we have $\nu(T, D) \geqslant \nu(\alpha, D)$, and $R+\sum(\nu(T, D)-\nu(\alpha, D))[D]$ is thus a Kähler current in $Z(\alpha)$ as desired.

(iv) Assume that $Z(\alpha)$ belongs to the interior $\mathcal{M N}^{0}$ of the modified nef cone. By Proposition 3.2, we have to see that $\nu(\alpha, D)=0$ for every prime $D$. Suppose therefore that $\nu\left(\alpha, D_{0}\right)>0$ for some prime $D_{0}$. The class $Z(\alpha)+\varepsilon\left\{D_{0}\right\}$ has to lie in the open cone $\mathcal{M N}^{0}$ for $\varepsilon$ small enough, thus we can write for $0<\varepsilon<\nu\left(\alpha, D_{0}\right)$ :

$$
\alpha=\left(Z(\alpha)+\varepsilon\left\{D_{0}\right\}\right)+\left(\nu\left(\alpha, D_{0}\right)-\varepsilon\right)\left\{D_{0}\right\}+\left\{\sum_{D \neq D_{0}} \nu(\alpha, D) D\right\} .
$$


We deduce that

$$
\nu\left(\alpha, D_{0}\right) \leqslant \nu\left(Z(\alpha)+\varepsilon\left\{D_{0}\right\}, D_{0}\right)+\left(\nu\left(\alpha, D_{0}\right)-\varepsilon\right) .
$$

Indeed, the class $\left\{D_{0}\right\}$ (respectively $\left\{\sum_{D \neq D_{0}} \nu(\alpha, D) D\right\}$ ) has minimal multiplicity $\leqslant 1$ (respectively 0 ) along $D_{0}$, because so is the generic Lelong numbers of the positive current $\left[D_{0}\right]$ (respectively $\left.\sum_{D \neq D_{0}} \nu(\alpha, D)[D]\right)$ along $D_{0}$. Now, we also have $\nu\left(Z(\alpha)+\varepsilon\left\{D_{0}\right\}, D_{0}\right)=0$ since $Z(\alpha)+\varepsilon\left\{D_{0}\right\}$ is modified nef by assumption, hence the contradiction $\nu\left(\alpha, D_{0}\right) \leqslant \nu\left(\alpha, D_{0}\right)-\varepsilon$.

Proposition 3.9.-(i) The map $\alpha \mapsto N(\alpha)$ is convex and homogeneous on $\mathcal{E}$. It is continuous on the interior of the pseudo-effective cone.

(ii) The Zariski projection $Z: \mathcal{E} \rightarrow \mathcal{M N}$ is concave and homogeneous. It is continuous on the interior of $\mathcal{E}$.

Proof. - We have already noticed that $\nu(\alpha+\beta, D) \leqslant \nu(\alpha, D)+\nu(\beta, D)$ for every prime $D$ and every two pseudo-effective classes $\alpha$, $\beta$. This implies that $N(\alpha+\beta) \leqslant N(\alpha)+N(\beta)$. Homogeneity is obvious, and the first assertion follows. To show continuity, it is enough as above to show that $\alpha \mapsto \int N(\alpha) \wedge \theta$ is continuous on $\mathcal{E}^{0}$ for every positive form $\theta$. But the latter map is convex, and thus continuous on $\mathcal{E}^{0}$ as any convex map on an open convex subset of a finite dimensional vector space is. (ii) is now an obvious consequence of (i) and the relation $Z(\alpha)=\alpha-\{N(\alpha)\}$.

\subsection{Negative part and exceptional divisors}

If $A=D_{1}, \ldots, D_{r}$ is a finite family of prime divisors, we denote by $V_{+}(A) \subset H_{\partial \bar{\partial}}^{1,1}(X, \mathbf{R})$ the closed convex cone generated by the classes $\left\{D_{1}\right\}, \ldots,\left\{D_{r}\right\}$. Every element of $V_{+}(A)$ writes $\alpha=\{E\}$ for some effective $\mathbf{R}$-divisor supported by the $D_{j}$ 's. Since $[E]$ is a positive current in $\alpha$, we have $N(\alpha) \leqslant E$, and thus $Z(\alpha)$ can be represented by the effective $\mathbf{R}$-divisor $E-N(\alpha)$, which is also supported by the $D_{j}$ 's. We conclude: $V_{+}(A)$ is stable under the Zariski projection $Z$. In particular, we have $Z\left(V_{+}(A)\right)=0$ iff $V_{+}(A)$ meets $\mathcal{M N}$ at 0 only.

Definition 3.10 (Exceptional divisors). - (i) A family $D_{1}, \ldots, D_{q}$ of prime divisors is said to be an exceptional family iff the convex cone generated by their cohomology classes meets the modified nef cone $\mathcal{M N}$ at 0 only.

(ii) An effective $\mathbf{R}$-divisor $E$ is said to be exceptional iff its prime components constitute an exceptional family.

We have the following

Proposition 3.11. - (i) An effective $\mathbf{R}$-divisor $E$ is exceptional iff $Z(\{E\})=0$.

(ii) If $E$ is an exceptional effective $\mathbf{R}$-divisor, we have $E=N(\{E\})$.

(iii) If $D_{1}, \ldots, D_{q}$ is an exceptional family of primes, then their classes $\left\{D_{1}\right\}, \ldots,\left\{D_{q}\right\}$ are linearly independent in $N S(X)_{\mathbf{R}} \subset H_{\partial \bar{\partial}}^{1,1}(X, \mathbf{R})$. In particular, the length of the exceptional families of primes is uniformly bounded by the Picard number $\rho(X)$.

Proof. - (i) Let $A=D_{1}, \ldots, D_{r}$ denote the family of primes supporting $E$, and choose a Gauduchon metric $\omega$ (cf. Section 2.2.4). Since $\omega^{n-1}$ is $\partial \bar{\partial}$-closed, $\int Z(\alpha) \wedge \omega^{n-1}$ is well defined, and defines a map $\mathcal{E} \rightarrow \mathbf{R} \alpha \mapsto \int Z(\alpha) \wedge \omega^{n-1}$, which is concave and homogeneous (by Proposition 3.11), and everywhere non-negative. The restriction of this map to $V_{+}(A)$ shares the same properties, and the class $\alpha:=\{E\}$ is a point in the relative interior of the convex cone $V_{+}(A)$ at which $\int Z(\alpha) \wedge \omega^{n-1}=0$. By concavity, we thus get $\int Z(\alpha) \wedge \omega^{n-1}=0$ for every $\alpha \in V_{+}(A)$, and thus $Z(\alpha)=0$ for every such $\alpha \in V_{+}(A)$. 
(ii) When $E$ is exceptional, we have both $E \geqslant N(\{E\})$ (because the positive current $[E]$ lies in the class $\{E\}$ ) and $\{E\}=\{N(\{E\})\}$ (because $Z(\{E\})=0$ ). Since a closed positive current which yields zero in $H_{\partial \bar{\partial}}^{1,1}(X, \mathbf{R})$ is itself zero, we get the result.

(iii) Since $D_{1}, \ldots, D_{q}$ are linearly independent in $\operatorname{Div}(X) \otimes \mathbf{R}$, the assertion is equivalent to the fact that the quotient map $D \mapsto\{D\}$ is injective on the $\mathbf{R}$-vector space of divisors generated by the $D_{j}$ 's. But this is easy: if $E=\sum a_{j} D_{j}$ lies in the kernel, we can write $E=E_{+}-E_{-}$with $E_{+}$and $E_{-}$effective such that $\left\{E_{+}\right\}=\left\{E_{-}\right\}$. By (iii), we get $E_{+}=E_{-}$, whence $E=0$.

We state as a theorem the following important consequences of (iii):

THEOREM 3.12. - (i) For every pseudo-effective class $\alpha \in \mathcal{E}$, the negative part $N(\alpha)$ is an exceptional effective $\mathbf{R}$-divisor supported by at most $\rho(X)$ primes.

(ii) $X$ carries at most countably many exceptional primes.

(iii) The exceptional fiber $Z^{-1}(0)$ is contained in $N S(X)_{\mathbf{R}}$, and is a union of at most countably many simplicial cones over exceptional families of primes.

Proof. - (i) We have $Z(\alpha) \geqslant Z(Z(\alpha))+Z(\{N(\alpha)\})$, and $Z(Z(\alpha))=Z(\alpha)$ by Proposition 3.10, thus $Z(\{N(\alpha)\})=0$. We immediately deduce from this that any family of primes $D_{1}, \ldots, D_{r}$ such that $\nu\left(\alpha, D_{j}\right)>0$ for every $j$ is an exceptional family, and the assertion follows from (iii) of Proposition 3.13.

(ii) We just have to notice that $D \mapsto\{D\}$ is injective on the set of exceptional primes, and maps into the lattice $N S(X) \subset N S(X)_{\mathbf{R}}$.

(iii) Since $\{A\}$ is a linearly independent set for every exceptional family of primes $A$, we see that $V_{+}(A)=\sum_{D \in A} \mathbf{R}_{+}\{D\}$ is a simplicial cone. It remains to observe that $\alpha$ lies in the exceptional fiber $Z^{-1}(0)$ iff $\alpha=\{N(\alpha)\}$, thus $Z^{-1}(0)$ is covered by the simplicial cones $V_{+}(A)$.

We will see in Section 4.3 that a family $D_{1}, \ldots, D_{q}$ of primes on a surface is exceptional iff the Gram matrix $\left(D_{i} \cdot D_{j}\right)$ is negative definite, i.e. iff $D_{1}, \ldots, D_{q}$ can all be blown down to points by a modification towards an analytic surface (singular in general). On a general compact complex $n$-fold $X$, an exceptional divisor is still very rigidly embedded in $X$ :

PROPOSITION 3.13. - If $E$ is an exceptional effective $\mathbf{R}$-divisor, then its class $\{E\}$ contains but one positive current, which is $[E]$. In particular, when $E$ is rational, its Kodaira-Iitaka dimension $\kappa(X, E)$ is zero.

Proof. - If $T$ is a positive current in $\{E\}$, we have $\nu(T, D) \geqslant \nu(\{E\}, D)$ for every prime $D$. Using the Siu decomposition of $T$, we thus see that $T \geqslant \sum \nu(\{E\}, D) D=N(\{E\})=E$, since $E$ is exceptional. But we also have $\{T\}=\{E\}$, hence $T=E$, as was to be shown. To get the last point, let $D$ be an element of the linear system $|k E|$ for some integer $k>0$ such that $k E$ is Cartier. The positive current $\frac{1}{k}[D]$ then lies in $\{E\}$, thus we have $[D]=k[E]$ as currents, hence $D=k E$ as divisors. This shows that $h^{0}(k E)=1$ for each $k>0$.

\subsection{Discontinuities of the Zariski projection}

It is remarkable that the Zariski projection $Z$ is not continuous in general up to the boundary $\partial \mathcal{E}$.

PROPOSITION 3.14. - If $X$ carries infinitely many exceptional primes, then the Zariski projection $Z: \mathcal{E} \rightarrow \mathcal{M N}$ is not continuous.

Proof. - We use the following

$4^{\mathrm{e}}$ SÉRIE - TOME $37-2004-\mathrm{N}^{\circ} 1$ 
LEMmA 3.15. - If $D_{k}$ is an infinite sequence of divisors, the rays $\mathbf{R}_{+}\left\{D_{k}\right\} \subset \mathcal{E}$ can accumulate on $\mathcal{M N}$ only.

Proof. - Suppose that $t_{k}\left\{D_{k}\right\}$ converges to some non-zero $\alpha \in \mathcal{E}$ (for $t_{k}>0$ ). For each prime $D$, we then have $D_{k} \neq D$ and thus $\nu\left(t_{k}\left\{D_{k}\right\}, D\right)=t_{k} \nu\left(\left\{D_{k}\right\}, D\right)=0$ for infinitely many $k$, because the family $D_{k}$ is infinite. By lower semi-continuity (Proposition 3.7) we deduce $\nu(\alpha, D)=0$ for every prime $D$, i.e. $\alpha$ is modified nef (by Proposition 3.2).

Assume now that an infinite sequence of exceptional prime divisors $D_{k}$ exists. Since $\mathcal{E}$ has a compact base, upon extracting a subsequence, we can assume that $t_{k}\left\{D_{k}\right\}$ converges to some non-zero $\alpha \in \mathcal{E}$ (with $t_{k}>0$ an appropriate sequence). Since $D_{k}$ is exceptional, we have $Z\left(t_{k}\left\{D_{k}\right\}\right)=0$ for every $k$, but $Z(\alpha)=\alpha$ since $\alpha$ is modified nef by the above lemma. Consequently, $Z^{-1}(0)$ is not closed, and $Z$ is not continuous.

To get an example of discontinuous Zariski projection, just take $X$ to be the blow-up of $\mathbf{P}^{2}$ in at least 9 general points. Such a rational surface is known to carry countably many exceptional curves of the first kind (cf. [13, p. 409]). Since a prime divisor $C$ on a surface is exceptional iff $C^{2}<0$ (cf. Section 4.3), the set of exceptional primes on $X$ is infinite, and we have our example.

\subsection{When is a decomposition the Zariski decomposition?}

Suppose that we have a decomposition $\alpha=p+\{N\}$ of a pseudo-effective class $\alpha$ into the sum of a modified nef class $p$ and the class of an effective $\mathbf{R}$-divisor $N$. We want a criterion that tells us when it is the Zariski decomposition of $\alpha$. We have $N(\alpha) \leqslant N(p)+N$, and $N(p)=0$ since $p$ is modified nef, thus $N(\alpha)=N$ happens iff $Z(\alpha)=p$, and our question is equivalent to the study of the fibers $Z^{-1}(p)$, with $p \in \mathcal{M N}$.

We will need the following

Definition 3.16 (Non-Kähler locus). - If $\alpha \in H_{\partial \bar{\partial}}^{1,1}(X, \mathbf{R})$ is a big class, we define its nonKähler locus as $E_{n K}(\alpha):=\bigcap_{T} E_{+}(T)$ for $T$ ranging among the Kähler currents in $\alpha$.

Let us explain the terminology:

THEOREM 3.17. - Let $\alpha \in H_{\partial \bar{\partial}}^{1,1}(X, \mathbf{R})$ be a big class. Then:

(i) The non-neflocus $E_{n n}(\alpha)$ is contained in the non-Kähler locus $E_{n K}(\alpha)$.

(ii) There exists a Kähler current with analytic singularities $T$ in $\alpha$ such that

$$
E_{+}(T)=E_{n K}(\alpha)
$$

In particular, the non-Kähler locus $E_{n K}(\alpha)$ is an analytic subset of $X$.

(iii) $\alpha$ is a Kähler class iff $E_{n K}(\alpha)$ is empty. More generally, $\alpha$ is a Kähler class iff $\alpha_{\mid Y}$ is a Kähler class for every irreducible component $Y$ of the analytic set $E_{n K}(\alpha)$.

Proof. - (i) Since $\alpha$ is big, its non-nef locus $E_{n n}(\alpha)$ is just the set $\left\{x \in X, \nu\left(T_{\min }, x\right)>0\right\}$, since we have $\nu(\alpha, x)=\nu\left(T_{\min }, x\right)$ in that case (cf. Proposition 3.8). For every Kähler current $T$ in $\alpha$, we have $\nu(T, x) \geqslant \nu\left(T_{\min }, x\right)$ by minimality, and the inclusion $E_{n n}(\alpha) \subset E_{n K}(\alpha)$ ensues.

(ii) First, we claim that given two Kähler currents $T_{1}, T_{2}$ in $\alpha$, there exists a Kähler current with analytic singularities $T$ such that $E_{+}(T) \subset E_{+}\left(T_{1}\right) \cap E_{+}\left(T_{2}\right)$. Indeed, we can find $\varepsilon>0$ small enough such that $T_{j} \geqslant \varepsilon \omega$. Our currents $T_{1}$ and $T_{2}$ thus belong to $\alpha[\varepsilon \omega]$, and admit an infimum $T_{3}$ in that set with respect to $\preceq$ (cf. Section 2.8). In particular, $T_{3}$ is a current in $\alpha$ with $T_{3} \geqslant \varepsilon \omega$ and $\nu\left(T_{3}, x\right)=\min \left\{\nu\left(T_{1}, x\right), \nu\left(T_{2}, x\right)\right\}$ for every $x \in X$. By (ii) of Theorem 2.1, there exists a Kähler current with analytic singularities $T$ in $\alpha$ such that $\nu(T, x) \leqslant \nu\left(T_{3}, x\right)$ for every $x \in X$, hence $E_{+}(T) \subset E_{+}\left(T_{1}\right) \cap E_{+}\left(T_{2}\right)$, and this proves the claim. 
Using the claim and (ii) of Theorem 2.1, it is easy to construct a sequence $T_{k}$ of Kähler currents with analytic singularities such that $E_{+}\left(T_{k}\right)$ is a decreasing sequence with

$$
E_{n K}(\alpha)=\bigcap_{k} E_{+}\left(T_{k}\right)
$$

Since $T_{k}$ has analytic singularities, $E_{+}\left(T_{k}\right)$ is an analytic subset, thus the decreasing sequence $E_{+}\left(T_{k}\right)$ has to be stationary (by the strong Nötherian property), and we eventually get $E_{n K}(\alpha)=E_{+}\left(T_{k}\right)$ for some $k$, as desired.

(iii) If $\alpha$ is a Kähler class, $E_{+}(\omega)$ is empty for every Kähler form $\omega$ in $\alpha$, and thus so is $E_{n K}(\alpha)$. Conversely, assume that $\alpha_{\mid Y}$ is a Kähler class for every component $Y$ of $E_{+}(\alpha)$, and let $T$ be a Kähler current with analytic singularities such that $E_{+}(T)=E_{n K}(\alpha)$. $\alpha$ is then a Kähler class by Proposition 3.3 of [7].

We can now state the following

THEOREM 3.18. - Let $p$ be a big and modified nef class. Then the primes $D_{1}, \ldots, D_{r}$ contained in the non-Kähler locus $E_{n K}(p)$ form an exceptional family $A$, and the fiber of $Z$ above $p$ is the simplicial cone $Z^{-1}(p)=p+V_{+}(A)$. When $p$ is an arbitrary modified nef class, $Z^{-1}(p)$ is an at most countable union of simplicial cones $p+V_{+}(A)$, where $A$ is an exceptional family of primes.

Proof. - Note that, by the very definitions, for every pseudo-effective class $\alpha$, the prime components of its negative part $N(\alpha)$ are exactly the set $A$ of primes $D$ contained in the nonnef locus $E_{n n}(\alpha)$. Furthermore, $Z(\alpha)+V_{+}(A)$ is entirely contained in the fiber $Z^{-1} Z(\alpha)$. Indeed, the restriction of $Z$ to this simplicial cone is a concave map above the affine constant map $Z(\alpha)$, and both coincide at the relative interior point $\alpha$, thus they are equal on the whole of $Z(\alpha)+V_{+}(A)$. This already proves the last assertion.

Assume now that $p$ is modified nef and big, and suppose first that $\alpha$ lies in $Z^{-1}(p)$. To see that $\alpha$ lies in $p+V_{+}(A)$, we have to prove that every prime $D_{0}$ with $\nu\left(\alpha, D_{0}\right)>0$ lies in $E_{n K}(p)$, that is: $\nu\left(T, D_{0}\right)>0$ for every Kähler current $T$ in $p$. If not, choose a smooth form $\theta$ in $\left\{D_{0}\right\}$. Since $T$ is a Kähler current, so is $T+\varepsilon \theta$ for $\varepsilon$ small enough. For $0<\varepsilon<\nu\left(\alpha, D_{0}\right)$ small enough, $T_{\varepsilon}:=T+\varepsilon \theta+\left(\nu\left(\alpha, D_{0}\right)-\varepsilon\right)\left[D_{0}\right]+\sum_{D \neq D_{0}} \nu(\alpha, D)[D]$ is then a positive current in $\alpha$ with $\nu\left(T_{\varepsilon}, D_{0}\right)=\nu\left(\alpha, D_{0}\right)-\varepsilon<\nu(\alpha, D)=\nu\left(T_{\min }, D_{0}\right)$ (the last equality holds by Proposition 3.8 because $\alpha$ is big since $p$ is); this is a contradiction which proves the inclusion $Z^{-1}(p) \subset p+V_{+}(A)$.

In the other direction, let $T$ be a Kähler current in $p$, and let $T=R+\sum \nu(T, D) D$ be its Siu decomposition. $R$ is then a Kähler current with $\nu(R, D)=0$ for every prime $D$, thus its class $\beta:=\{R\}$ is a modified Kähler class. We first claim that we have $D_{j} \subset E_{n n}(p-\varepsilon \beta)$ for every $\varepsilon>0$ small enough and every prime component $D_{j}$ of the non-Kähler locus $E_{n K}(p)$ of $p$. Indeed, since $p-\varepsilon \beta$ is big for $\varepsilon>0$ small enough, we have $\nu\left(p-\varepsilon \beta, D_{j}\right)=\nu\left(T, D_{j}\right)$ if $T$ is a positive current with minimal singularities in $p-\varepsilon \beta$, and we have to see that $\nu\left(T, D_{j}\right)>0$. But $T+\varepsilon R$ is a Kähler current in $p$, thus $D_{j} \subset E_{n K}(p) \subset E_{+}(T+\varepsilon R)$ by definition, which exactly means that $\nu\left(T+\varepsilon R, D_{j}\right)>0$. The claim follows since $\nu\left(R, D_{j}\right)=0$ by construction of $R$.

As a consequence of this claim, each prime $D_{1}, \ldots, D_{r}$ of our family $A$ occurs in the negative part $N(p-\varepsilon \beta)$ for $\varepsilon>0$ small enough. Consequently, by the first part of the proof, the Zariski projection of $Z(p-\varepsilon \beta)+\{E\}$ is just $Z(p-\varepsilon \beta)$ for every effective $\mathbf{R}$-divisor $E$ supported by the $D_{j}$ 's and every $\varepsilon>0$ small enough. Since $p$ is big, $Z$ is continuous at $p$, thus $Z(p-\varepsilon \beta)$ converges to $Z(p)$, which is just $p$ because the latter is also modified nef. Finally, $Z$ is also continuous at the big class $p+\{E\}$, thus the Zariski projection of $Z(p-\varepsilon \beta)+\{E\}$ converges 
to that of $p+\{E\}$, and thus $Z(p+\{E\})=p$ holds. This means that $p+V_{+}(A) \subset Z^{-1}(p)$, and concludes the proof of Theorem 3.20.

\subsection{Structure of the pseudo-effective cone}

Using our constructions, we will prove the

THEOREM 3.19. - The boundary of the pseudo-effective cone is locally polyhedral away from the modified nef cone, with extremal rays generated by (the classes of) exceptional prime divisors.

Proof. - This is in fact rather straightforward by now: for each prime $D$, the set

$$
\mathcal{E}_{D}:=\{\alpha \in \mathcal{E}, \nu(\alpha, D)=0\}
$$

is a closed convex subcone of $\mathcal{E}$. This follows from the fact that $\alpha \mapsto \nu(\alpha, D)$ is convex, homogeneous, lower semi-continuous and everywhere non-negative. If $\alpha \in \partial \mathcal{E}$ does not belong to $\mathcal{M N}$, it does not belong to $\mathcal{E}_{D}$ for some prime $D$ by Proposition 3.2. For every $\beta \in \mathcal{E}$, we have either $\beta \in \mathcal{E}_{D}$, or $D$ occurs in the negative part $N(\beta)$. Therefore, $\mathcal{E}$ is generated by $\mathbf{R}_{+}\{D\}$ and $\mathcal{E}_{D}$, and the latter does not contain $\alpha$. This means that $\partial \mathcal{E}$ is locally polyhedral near $\alpha$. Since $\nu(\alpha, D)>0$, we also see that $D$ is exceptional. Finally, the extremal rays of $\mathcal{E}$ not contained in $\mathcal{M N}=\bigcap_{D} \mathcal{E}_{D}$ have to lie outside $\mathcal{E}_{D}$ for some exceptional prime $D$, and since $\mathcal{E}=\mathcal{E}_{D}+\mathbf{R}_{+}\{D\}$, each such extremal ray is generated by $\{D\}$ for some $D$.

\subsection{Volumes}

Recall that the volume of a pseudo-effective class $\alpha$ on a compact Kähler $n$-fold is defined to be the supremum $v(\alpha)$ of $\int_{X} T_{a c}^{n}$ for $T$ a closed positive (1,1)-current in $\alpha$ (cf. [2]). A class $\alpha$ is big iff $v(\alpha)>0$, and the volume is a quantitative measure of its bigness. We have already noticed that $Z(\alpha)$ is big iff $\alpha$ is; we have the following quantitative version:

Proposition 3.20. - Let $\alpha$ be a pseudo-effective class on $X$ compact Kähler. Then $v(Z(\alpha))=v(\alpha)$.

The proof is in fact immediate: if $T$ is a positive current in $\alpha$, then we have $T \geqslant N(\alpha)$ since $T$ belongs to $\alpha[-\varepsilon \omega]$ for each $\varepsilon>0$, and we deduce that $T \rightarrow T-N(\alpha)$ is a bijection between the positive currents in $\alpha$ and those in $Z(\alpha)$. It remains to notice that $(T-N(\alpha))_{a c}=T_{a c}$ to conclude the proof.

\section{Zariski decomposition on a surface and a hyper-Kähler manifold}

It is known since the pioneering work of Zariski [19] that any effective divisor $D$ on a projective surface admits a unique Zariski decomposition $D=P+N$, i.e. a decomposition into a sum of $\mathbf{Q}$-divisors $P$ and $N$ with the following properties:

(i) $P$ is nef, $N=\sum a_{j} N_{j}$ is effective,

(ii) $P \cdot N=0$,

(iii) the Gram matrix $\left(N_{i} \cdot N_{j}\right)$ is negative definite.

We want to show that our divisorial Zariski decomposition indeed is a generalization of such a Zariski decomposition on a surface. 


\subsection{Notations}

$X$ will stand for a compact Kähler surface, or a compact hyper-Kähler manifold. For such an $X$, we denote by $q$ the quadratic form on $H^{1,1}(X, \mathbf{R})$ defined as follows: when $X$ is a surface, we set $q(\alpha):=\int \alpha^{2}$, and when $X$ is hyper-Kähler, we choose a symplectic holomorphic form $\sigma$, and let $q(\alpha):=\int \alpha^{2}(\sigma \bar{\sigma})^{m-1}$ be the usual Beauville-Bogomolov quadratic form, with $\sigma$ normalized so as to achieve $q(\alpha)^{m}=\int_{X} \alpha^{2 m}$ (with $\operatorname{dim} X=n=2 m$ ). In both cases $\left(H^{1,1}(X, \mathbf{R}), q\right)$ is Lorentzian, i.e. it has signature $\left(1, h^{1,1}(X)-1\right)$; the open cone $\left\{\alpha \in H^{1,1}(X, \mathbf{R}), q(\alpha)>0\right\}$ has thus two connected components which are convex cones, and we denote by $\mathcal{P}$ the component containing the Kähler cone $\mathcal{K}$. We call $\mathcal{P}$ the positive cone (attached to the quadratic form $q$ ). In general, given a linear form $\lambda$ on $H^{1,1}(X, \mathbf{R})$, we will denote its kernel by $\lambda^{\perp}$ and the two open half-spaces it defines by $\lambda_{>0}$ and $\lambda_{<0}$. The dual $\mathcal{C}^{\star}$ of a convex cone $\mathcal{C}$ in $H^{1,1}(X, \mathbf{R})$ is seen as a cone in $H^{1,1}(X, \mathbf{R})$, using the duality induced by $q$.

\subsection{The dual pseudo-effective cone}

In both cases, we shall prove that the modified nef cone is the dual cone to the pseudo-effective cone.

\subsubsection{The case of a surface}

We suppose that $X$ is a surface. We prove the following essentially well-known

THEOREM 4.1. - When $X$ is a surface, the Kähler cone and the modified Kähler cone coincide. The dual pseudo-effective cone is just the nef cone.

Proof. - If $\alpha \in \mathcal{M K}$, it can be represented by a Kähler current with analytic singularities in codimension 2, that is at some points $x_{1}, \ldots, x_{r}$. Therefore we see that the non-Kähler locus $E_{n K}(\alpha)$ is a discrete set. Since the restriction of any class to a point is (by convention) a Kähler class, Theorem 3.19 shows that $\alpha$ lies in fact in $\mathcal{K}$.

Since $\int_{X} \omega \wedge T$ is positive for every Kähler form $\omega$ and every positive current $T$, we of course have $\mathcal{K} \subset \mathcal{E}^{\star}$, and thus also $\mathcal{N}=\bar{K} \subset \mathcal{E}^{\star}$. The other inclusion is much deeper, since it is a consequence of the Nakai-Moishezon criterion for Kähler classes on a surface, as given in [15]. Indeed, this criterion implies that a real $(1,1)$-class $\alpha$ on a Kähler surface is a nef class iff $\alpha \cdot \omega \geqslant 0$ for every $\omega \in \mathcal{K}$ and $\alpha \cdot C \geqslant 0$ for every irreducible curve $C$. Since a class in $\mathcal{E}^{\star}$ clearly satisfies these conditions, we get $\mathcal{E}^{\star} \subset \mathcal{N}$, and the proof of Theorem 4.1 is over.

As a consequence, since $\mathcal{K}$ is contained in $\mathcal{P}$ and since $\overline{\mathcal{P}}$ is self dual (just because $q$ is Lorentzian), we get dually that $\overline{\mathcal{P}} \subset \mathcal{E}$ and thus that $\mathcal{P} \subset \mathcal{E}^{0}=\mathcal{B}$, which means the following: if $\alpha$ is a real $(1,1)$-class with $\alpha^{2}>0$, then $\alpha$ or $-\alpha$ is big. This generalizes the well known case where $\alpha$ is (the first Chern class of) a line bundle (whose proof is based on Riemann-Roch).

\subsubsection{The hyper-Kähler case}

In that case, the dual pseudo-effective cone is also equal to the modified nef cone, but the proof uses another description, due to D. Huybrechts, of the dual pseudo-effective cone. In the easy direction, we have:

Proposition 4.2.-(i) The modified nef cone $\mathcal{M N}$ is contained in both the dual pseudoeffective cone $\mathcal{E}^{\star}$ and the closure of the positive cone $\overline{\mathcal{P}}$.

(ii) We have $q\left(D, D^{\prime}\right) \geqslant 0$ for any two distinct prime divisors $D \neq D^{\prime}$.

Proof. - To prove (i), we only have to prove that $\mathcal{M K} \subset \mathcal{E}^{\star}$. Indeed, $\mathcal{M K} \cap \mathcal{E}^{\star} \subset \mathcal{E} \cap \mathcal{E}^{\star}$ is trivially contained in $\overline{\mathcal{P}}$. We pick a modified Kähler class $\alpha$ and a pseudo-effective class $\beta \in \mathcal{E}$, 
and choose a Kähler current $T$ in $\alpha$ with analytic singularities in codimension at least 2, and a positive current $S$ in $\beta$. By Section 2.6, the wedge product $T \wedge S$ is well defined as a closed positive $(2,2)$-current, and lies in the class $\alpha \cdot \beta$. Since $(\sigma \bar{\sigma})^{m-1}$ is a smooth positive form of bidimension $(2,2)$, the integral $\int_{X} T \wedge S \wedge(\sigma \bar{\sigma})^{m-1}$ is positive. But $(\sigma \bar{\sigma})^{m-1}$ is also closed, thus we have

$$
\int_{X} T \wedge S \wedge(\sigma \bar{\sigma})^{m-1}=\alpha \cdot \beta \cdot\left\{(\sigma \bar{\sigma})^{m-1}\right\}=q(\alpha, \beta),
$$

so we have proven that $q(\alpha, \beta) \geqslant 0$ as desired.

The second contention is obtained similarly, noting that $\{D\} \cdot\left\{D^{\prime}\right\}$ contains a closed positive $(2,2)$-current, which is $\left[D \cdot D^{\prime}\right]$, where $D \cdot D^{\prime}$ is the effective intersection cycle.

The other direction $\mathcal{E}^{\star} \subset \mathcal{M N}$ is much deeper. The effective 1-dimensional cycles $C$ and the effective divisors $D$ define linear forms on $H^{1,1}(X, \mathbf{R})$ via the intersection form and the Beauville-Bogomolov form $q$ respectively, and we define a rational (respectively uniruled) chamber of the positive cone $\mathcal{P}$ to be a connected component of $\mathcal{P}-\bigcup C^{\perp}$ (respectively $\mathcal{P}-\bigcup D^{\perp}$ ), where $C$ (respectively $D$ ) runs over the rational curves (respectively the uniruled divisors). By a rational curve (respectively a uniruled divisor) we mean an effective 1-dimensional cycle all of whose components are irreducible rational curves (respectively an effective divisor all of whose components are uniruled prime divisors). The rational chamber of $\mathcal{P}$ cut out by all the $C_{>0}$ 's (respectively $D_{>0}$ 's) will be called the fundamental rational chamber (respectively the fundamental uniruled chamber). When $X$ is a $K 3$ surface, the rational and uniruled chambers are the same thing and coincide with the traditional chambers in that situation. We can now state the following fundamental result:

THEOREM 4.3 [14]. - (i) The positive cone $\mathcal{P}$ is contained in $\mathcal{E}$.

(ii) If $\alpha \in \mathcal{P}$ belongs to one of the rational chambers, then there exists a bimeromorphic map $f: X-\rightarrow X^{\prime}$ to a hyper-Kähler $X^{\prime}$ such that

$$
f_{\star} \alpha=\omega^{\prime}+\left\{D^{\prime}\right\},
$$

where $\omega^{\prime} \in \mathcal{K}_{X^{\prime}}$ is a Kähler class and $D^{\prime}$ is a uniruled $\mathbf{R}$-divisor.

(iii) When $\alpha \in \mathcal{P}$ lies in both the fundamental uniruled chamber and one of the rational chambers, then no uniruled divisor $D^{\prime}$ occurs in (ii).

(iv) The fundamental rational chamber coincides with the Kähler cone of $X$.

In fact, [14] states this only for a very general element $\alpha \in \mathcal{P}$, but we have noticed in [1] that the elements of the rational chambers are already very general in that respect.

In the situation (iii), $\alpha$ lies in $f^{\star} \mathcal{K}_{X^{\prime}}$ for some bimeromorphic $f: X-\rightarrow X^{\prime}$ towards a hyperKähler $X^{\prime}$. The union of such open convex cones $\mathcal{K}_{f}:=f^{\star} \mathcal{K}_{X^{\prime}}$ is called the bimeromorphic Kähler cone, and is denoted by $\mathcal{B K}$. The union in question yields in fact a partition of $\mathcal{B K}$ into open convex cones $\mathcal{K}_{f}$ (since a bimeromorphic map between minimal manifolds which sends one Kähler class to a Kähler class is an isomorphism by a result of A. Fujiki); $\mathcal{B K}$ is an open cone, but definitely not convex in general. (iii) tells us that each intersection of a rational chamber with the fundamental uniruled chamber is contained in $\mathcal{B K}$, and thus in one of the $\mathcal{K}_{f}$ 's.

We can now describe the dual pseudo-effective cone:

PROPOSITION 4.4. - The dual pseudo-effective $\mathcal{E}^{\star}$ of a hyper-Kähler manifold coincides with the modified nef cone $\mathcal{M N}$.

Proof. - By Proposition 4.2, it remains to see that $\mathcal{E}^{\star}$ is contained in the modified nef cone $\mathcal{M N}$. By (i) of Theorem 4.3, we have $\mathcal{E}^{\star} \subset \overline{\mathcal{P}}$, and it will thus be enough to show that an 
element of the interior of $\mathcal{E}^{\star}$ which belongs to one of the rational chambers lies in $\mathcal{M N}$. But an element $\alpha$ of the interior of $\mathcal{E}^{\star}$ has $q(\alpha, D)>0$ for every prime $D$, thus it certainly lies in the fundamental uniruled chamber. If $\alpha$ lies in both the interior of $\mathcal{E}^{\star}$ and one of the rational chambers, it therefore lies in $\mathcal{K}_{f}=f^{\star} \mathcal{K}_{X^{\prime}}$ for some bimeromorphic $f: X-\rightarrow X^{\prime}$, and it remains to see that $\mathcal{K}_{f} \subset \mathcal{M N}$. But if $\omega$ is a Kähler form on $X^{\prime}$, its pull-back $T:=f^{\star} \omega$ can be defined using a resolution of $f$, and it is easy to check that $T$ is a Kähler current with $\nu(T, D)=0$ for every prime $D$, since $f$ induces an isomorphism $X-A \rightarrow X^{\prime}-A^{\prime}$ for $A, A^{\prime}$ analytic subsets of codimension at least 2 (this is because $X$ and $X^{\prime}$ are minimal). Therefore, $\{T\}=f^{\star}\{\omega\}$ belongs to $\mathcal{M K} \subset \mathcal{M N}$.

\subsection{Exceptional divisors}

When $X$ is a surface or a hyper-Kähler manifold, the fact that a family $D_{1}, \ldots, D_{r}$ of prime divisors is exceptional can be read off its Gram matrix.

THEOREM 4.5. - A family $D_{1}, \ldots, D_{r}$ of prime divisors is exceptional iff its Gram matrix $\left(q\left(D_{i}, D_{j}\right)\right)$ is negative definite.

Proof. - Let $V$ (respectively $V_{+}$) be the real vector space of $\mathbf{R}$-divisors (respectively effective R-divisors) supported by the $D_{j}$ 's. We begin with a lemma of quadratic algebra:

Lemma 4.6. - Assume that $(V, q)$ is negative definite. Then every $E \in V$ such that $q\left(E, D_{j}\right) \leqslant 0$ for all $j$ belongs to $V_{+}$.

Proof. - If $E \in V$ is non-positive against each $D_{j}$, we write $E=E_{+}-E_{-}$where $E_{+}$and $E_{-}$are effective with disjoint supports. We have to prove that $E_{-}=0$, and this is equivalent by assumption to $q\left(E_{-}\right) \geqslant 0$. But $q\left(E_{-}\right)=q\left(E_{-}, E_{+}\right)-q\left(E_{-}, E\right)$. The first term is positive because $E_{+}$and $E_{-}$have disjoint supports, using (ii) of Proposition 4.2, whereas the second is positive by assumption on $E$.

Let $D_{1}, \ldots, D_{r}$ be primes with negative definite Gram matrix. In particular, we then have that $\left\{V_{+}\right\} \subset H^{1,1}(X, \mathbf{R})$ meets $\overline{\mathcal{P}}$ at 0 only. Since the modified nef cone $\mathcal{M N}$ is contained in $\overline{\mathcal{P}}$ by Proposition 4.2, $\left\{V_{+}\right\}$a fortiori meets the modified nef cone at 0 only, which means by definition that $D_{1}, \ldots, D_{r}$ is an exceptional family, and this proves necessity in Theorem 4.5. In the other direction, assume that $D_{1}, \ldots, D_{r}$ is an exceptional family of primes. We first prove that the matrix $\left(q\left(D_{i}, D_{j}\right)\right)$ is semi-negative. If not, we find an R-divisor $E$ in $V$ with $q(E)>0$. Writing again $E=E_{+}-E_{-}$, with $E_{+}$and $E_{-}$two effective divisors in $V_{+}$with disjoint supports, we have again $q\left(E_{+}, E_{-}\right) \geqslant 0$ by (ii) of Proposition 4.2 , and thus $q\left(E_{+}\right)+q\left(E_{-}\right) \geqslant q(E)>0$. We may therefore assume that $E$ lies in $V_{+}$, with $q(E)>0$. But then $E$ or $-E$ is big, and it has to be $E$ because it is already effective. Its Zariski projection $Z(\{E\})$ is then non-zero since it is also big (by Proposition 3.10), and it lies in both $\left\{V_{+}\right\}$and $\mathcal{M N}$, a contradiction.

To conclude the proof of Theorem 4.5, we may assume (by induction) that the Gram matrix of $D_{1}, \ldots, D_{r-1}$ is negative definite. If $(V, q)$ is degenerate, the span $V^{\prime}$ of $D_{1}, \ldots, D_{r-1}$ is such that its orthogonal space $V^{\prime \perp}$ in $V$ is equal to the null-space of $V$. We then decompose $D_{r}=E+F$ in the direct sum $V=V^{\prime} \oplus V^{\prime \perp}$. Since $q\left(E, D_{j}\right)=q\left(D_{r}, D_{j}\right) \geqslant 0$ for $j<r$, Lemma 4.6 yields that $E \leqslant 0$. Therefore, $F=D_{r}-E$ lies in $V_{+}$, and is certainly non-zero. We claim that $\{F\}$ is also modified nef, which will yield the expected contradiction. But $F$ lies in the null-space of $V$, and is therefore non-negative against every prime divisor $D$. If $\alpha$ is a pseudoeffective class, we have $q(\{F\}, \alpha)=q(\{F\}, Z(\alpha))+q(F, N(\alpha))$. The first term is positive since $Z(\alpha) \in \mathcal{M N}=\mathcal{E}^{\star}$, and the second one is positive because $F$ is positive against every effective divisor. We infer from all this that $\{F\}$ lies in $\mathcal{E}^{\star}=\mathcal{M N}$, and the claim follows. 
The theorem says in particular that a prime divisor $D$ is negative iff $q(D)<0$. On a $\mathrm{K} 3$ surface, an easy and well-known argument using the adjunction formula shows that the prime divisors with negative square are necessarily smooth rational curves with square -2 . In higher dimension, we have:

PROPOSITION 4.7.-On a hyper-Kähler manifold $X$, the exceptional prime divisors are uniruled.

Proof. - Since $D$ is exceptional, it lies outside $\overline{\mathcal{P}}=\mathcal{P}^{\star}$, and we thus find a class $\alpha \in \mathcal{P}$ lying in one of the rational chambers such that $q(\alpha, D)<0$. By (ii) of Theorem 4.3, there exists a bimeromorphic map between hyper-Kähler manifolds $f: X-\rightarrow X^{\prime}$ such that $f_{\star} \alpha=\omega^{\prime}+\sum a_{j} D_{j}^{\prime}$ with $\omega^{\prime}$ a Kähler class, $a_{j} \geqslant 0$ and $D_{j}^{\prime}$ a uniruled prime divisor. Since the quadratic form is preserved by $f$, we have $0>q(\alpha, D)=q\left(\omega^{\prime}, f_{\star} D\right)+\sum a_{j} q\left(D_{j}^{\prime}, f_{\star} D\right)$, and $q\left(D_{j}^{\prime}, f_{\star} D\right)$ has to be negative for some $j$. But this implies that the two primes $D_{j}^{\prime}$ and $f_{\star} D$ coincide, and thus $D=f^{\star} D_{j}^{\prime}$ is uniruled since $D_{j}^{\prime}$ is.

\subsection{Rationality of the Zariski decomposition}

We want to prove that the divisorial Zariski decomposition is rational (when $X$ is a surface or a hyper-Kähler manifold) in the sense that $N(\alpha)$ is a rational divisor when $\alpha$ is a rational class. We first show the following characterization of the divisorial Zariski decomposition:

THEOREM 4.8. - If $\alpha \in H^{1,1}(X, \mathbf{R})$ is a pseudo-effective class, its divisorial Zariski decomposition $\alpha=Z(\alpha)+\{N(\alpha)\}$ is the unique orthogonal decomposition of $\alpha$ into the sum of a modified nef class and the class of an exceptional effective $\mathbf{R}$-divisor.

Proof. - We first prove uniqueness: assume that $\alpha=p+\{N\}$ is an orthogonal decomposition with $p$ a modified nef class and $N$ an effective exceptional $\mathbf{R}$-divisor. We claim that $N(\alpha)=N$. To see this, let $D_{1}, \ldots, D_{r}$ be the support of $N$; the Gram matrix $\left(q\left(D_{i}, D_{j}\right)\right)$ is negative definite by Theorem 4.5, and $p$ is orthogonal to each $D_{j}$ because $q(p, N)=0$ and $q\left(p, D_{j}\right) \geqslant 0$ for all $j$ since $p$ is a modified nef class. We have $N(\alpha) \leqslant N(p)+N$ and $N(p)=0$ since $p$ is modified nef, thus $N(\alpha) \leqslant N$. But $N(\alpha)-N$ is supported by primes $D_{1}, \ldots, D_{r}$ whose Gram matrix is negative definite, and $q\left(N(\alpha)-N, D_{j}\right)=q\left(p, D_{j}\right)-q\left(Z(\alpha), D_{j}\right)$ is non-positive since $p$ is orthogonal to $D_{j}$ and $Z(\alpha)$ belongs to $\mathcal{M N}=\mathcal{E}^{\star}$. Lemma 4.6 thus yields $N(\alpha) \geqslant N$, and the claim follows. To prove Theorem 4.8, we will show the existence of an orthogonal decomposition $\alpha=p+\{N\}$ with $p$ a modified nef class and $N$ an exceptional $\mathbf{R}$-divisor. When this is done, we must have $N=N(\alpha)$ by the claim, so that $\alpha=Z(\alpha)+\{N(\alpha)\}$ is itself an orthogonal decomposition.

LEMmA 4.9. - A pseudo-effective class $\alpha$ lies in $\mathcal{E}^{\star}$ iff $q(\alpha, D) \geqslant 0$ for every prime $D$.

Proof. - If $\beta$ is a pseudo-effective class, we write $q(\alpha, \beta)=q(\alpha, Z(\beta))+q(\alpha, N(\beta))$. The first term is positive because $Z(\beta)$ lies in $\mathcal{E}^{\star}$, and the second one is positive if $q(\alpha, D) \geqslant 0$ for each prime $D$.

LemMA 4.10. - Let $\alpha$ be a pseudo-effective class and let $D_{1}, \ldots, D_{r}, E_{1}, \ldots, E_{p}$ be two families of primes such that:

(i) $q\left(\alpha, D_{j}\right)<0$ and $q\left(\alpha, E_{i}\right) \leqslant 0$ for every $j$ and $i$.

(ii) $E_{1}, \ldots, E_{r}$ is an exceptional family.

Then the union of these two families is exceptional.

Proof. - Let $F$ be an effective divisor supported by $D_{j}$ 's and $E_{i}$ 's, and assume that $\{F\}$ is a modified nef class. We have to see that $F=0$. But $q(\alpha, F)$ is positive since $F$ is modified nef, 
thus we see using (i) that $F$ is in fact supported by $E_{i}$ 's, and then (ii) enables us to conclude that $F=0$ as desired.

At this point, the argument is similar to [11]. If the pseudo-effective class $\alpha$ is already in $\mathcal{E}^{\star}$, we trivially have our decomposition. Otherwise, consider the family $A$ of primes $D$ such that $q(\alpha, D)<0$. That family is exceptional by Lemma 4.10 with $E_{1}, \ldots, E_{p}$ an empty family, thus $A$ is finite with negative definite Gram matrix, and is non-empty by Lemma 4.9. Let

$$
\alpha=\alpha_{1}+\left\{N_{1}\right\}
$$

be the decomposition in the direct sum $V^{\perp} \oplus V$, where $V \subset H^{1,1}(X, \mathbf{R})$ is spanned by $A$. We claim that $N_{1}$ is effective and that $\alpha_{1}$ is pseudo-effective. Since $q\left(N_{1}, D\right)=q(\alpha, D)<0$ for every $D \in A$, Lemma 4.6 yields that $N_{1}$ is effective. We can also write $N(\alpha)=E+F$ where $E$ and $F$ are effective with disjoint supports and $F$ is supported by elements of $A$. Then for every $D \in A$ we have $q\left(F-N_{1}, D\right) \leqslant q\left(N(\alpha)-N_{1}, D\right)$ since $E$ and $D$ are disjoint, and $q\left(N(\alpha)-N_{1}, D\right)=q\left(\alpha_{1}, D\right)-q(Z(\alpha), D)$ is non-positive because $\alpha_{1}$ and $D$ are orthogonal and $Z(\alpha)$ lies in $\mathcal{E}^{\star}$. We infer from this that $N(\alpha) \geqslant N_{1}$ using Lemma 4.6, and $\alpha_{1}=Z(\alpha)+\left\{N(\alpha)-N_{1}\right\}$ is thus pseudo-effective, and this proves our claim.

If $\alpha_{1}$ lies in $\mathcal{E}^{\star}$, we have our decomposition by construction; otherwise, we iterate the construction: let $B$ be the non-empty exceptional family of primes $D$ such that $q\left(\alpha_{1}, D\right)<0$. Since $A$ is already exceptional and $q\left(\alpha_{1}, D\right)=0$ for $D \in A$, we infer from Lemma 4.10 that the union $A_{1}$ of $A$ and $B$ is again an exceptional family. We decompose

$$
\alpha_{1}=\alpha_{2}+\left\{N_{2}\right\}
$$

in the direct sum $V_{1}^{\perp} \oplus V_{1}$, where $V_{1} \subset H^{1,1}(X, \mathbf{R})$ is spanned by $A_{1}$. The same arguments as above show in that case also that $\alpha_{2}$ is pseudo-effective, and also that $N_{2}$ is effective (since $q\left(N_{2}, D\right)=q\left(\alpha_{1}, D\right) \leqslant 0$ for each $\left.D \in A_{1}\right)$. But since $B$ is non-empty, $A_{1}$ is an exceptional family strictly bigger than $A$. Since the length of the exceptional families is uniformly bounded by the Picard number $\rho(X)$ by Theorem 3.14, the iteration of the construction has to stop after $l$ steps, for which we get a class $\alpha_{l}$ which is modified nef. The desired decomposition is then obtained by setting $p:=\alpha_{l}$ and $N:=N_{1}+\cdots+N_{l}$, which is exceptional since it is supported by elements of $A \cup A_{1} \cup \cdots \cup A_{l}=A_{l}$ (since $A \subset A_{1} \subset \cdots \subset A_{1}$ by construction). This concludes the proof of Theorem 4.8 .

COROLlary 4.11 (Rationality of the Zariski decomposition). - The divisorial Zariski decomposition is rational in case $X$ is a surface or a hyper-Kähler manifold. In particular, when $D$ is a pseudo-effective divisor on $X$, the modified nef $\mathbf{R}$-divisor $P:=D-N(\{D\})$ is rational and such that the canonical inclusion of $H^{0}(X, \mathcal{O}(k P))$ in $H^{0}(X, \mathcal{O}(k D))$ is surjective for every $k$ such that $k P$ is Cartier.

Proof. - If $\alpha \in N S(X) \otimes \mathbf{Q}$ is a rational class, $N(\alpha)$ is necessarily the image of $\alpha$ by the orthogonal projection $N S(X) \otimes \mathbf{Q} \rightarrow V_{\mathbf{Q}}(\alpha)$, where $V_{\mathbf{Q}}(\alpha)$ is the $\mathbf{Q}$-vector space generated by the cohomology classes of the components of $N(\alpha)$. The latter is therefore rational. As to the second part, let $E$ be an element of the linear system $|k D|$. Since the integration current $\frac{1}{k}[E]$ is positive and lies in $\{D\}$, we have $E \geqslant k N(\{D\})$. But this exactly means that $k N(\{D\})$ is contained in the base scheme of $|k D|$, as was to be shown.

Proposition 4.12 (Rationality of the volume). - If $p \in H^{1,1}(X, \mathbf{R})$ is a modified nef class on $X$, its volume is equal to

$$
v(p)=q(p)^{m}=\int p^{\operatorname{dim} X}
$$


In general, we have $v(\alpha)=\int Z(\alpha)^{\operatorname{dim} X}$; in particular, the volume of a rational class is rational.

Proof. - We have already proven in Proposition 3.22 that $v(\alpha)=v(Z(\alpha))$, so only the first assertion needs a proof. We have shown in [2] that the equality $v(p)=\int p^{\operatorname{dim} X}$ is always true when $p$ is a nef class, so the contended equality holds on a surface. In the hyper-Kähler case, since we have chosen the symplectic form $\sigma$ so that $q(\alpha)^{m}=\alpha^{2 m}$ for any class $\alpha$, we just have to prove $v(p)=q(p)^{m}$ for $p \in \mathcal{M N}$. The latter cone is also the closure of the bimeromorphic Kähler cone $\mathcal{B K}$, so we may assume that $p$ lies in $f^{\star} \mathcal{K}_{X^{\prime}}$ for some bimeromorphic map $f: X-\rightarrow X^{\prime}$ between hyper-Kähler manifolds (because both $q$ and the volume are continuous). But since $f$ is an isomorphism in codimension 1 , the volume is invariant under $f$, and so is the quadratic form $q$, so we are reduced to the case where $p$ is a Kähler class, for which the equality is always true as we have said above.

\section{The algebraic approach}

In this section, we would like to show what the constructions we have made become when $\alpha=c_{1}(L)$ is the first Chern class of a line bundle on a projective complex manifold $X$. The general philosophy is that the divisorial Zariski decomposition of a big line bundle can be defined algebraically in terms of the asymptotic linear series $|k L|$. When $L$ is just pseudo-effective, sections are of course not sufficient, but we are led back to the big case by approximating. For those who are reluctant to assume projectivity too quickly, we remark that a compact Kähler manifold carrying a big line bundle is automatically projective.

\subsection{From sections to currents and back}

Let $L \rightarrow X$ be a line bundle over the projective manifold $X$. Each time $L$ has sections $\sigma_{1}, \ldots, \sigma_{l} \in H^{0}(X, L)$, there is a canonical way to construct a closed positive current $T \in c_{1}(L)$ with analytic singularities as follows: choose some smooth Hermitian metric $h$ on $L$, and consider

$$
\varphi(x):=\frac{1}{2} \log \sum_{j} h\left(\sigma_{j}(x)\right) .
$$

Then we define $T=\Theta_{h}(L)+d d^{c} \varphi$, where $\Theta_{h}(L)$ is the first Chern form of $h$. One immediately checks that $T$ is positive and independent of the choice of $h$, and thus depends on the sections $\sigma_{j}$ only. $T$ has analytic singularities exactly along the common zero-scheme $A$ of the $\sigma_{j}$ 's, and its Siu decomposition therefore writes $T=R+D$, where $D$ is the divisor part of $A$. When $\left(\sigma_{j}\right)$ is a basis of $H^{0}(X, L)$, we set $T_{|L|}:=T$. Another way to see $T_{|L|}$ is as the pull-back of the FubinyStudy form on $\mathbf{P} H^{0}(X, L)^{\star}=\mathbf{P}^{N}$ (the identification is determined by the choice of the basis of $\left.H^{0}(L)\right)$ by the rational map $\phi_{|L|}: X-\rightarrow \mathbf{P} H^{0}(X, L)^{\star} . T_{|L|}$ is independent of the choice of the basis up to equivalence of singularities, and carries a great deal of information about the linear system $|L|$ : the singular scheme $A$ of $T_{|L|}$ is the base scheme $B_{|L|}$ of the linear system $|L|$, the Lelong number $\nu\left(T_{|L|}, x\right)$ at $x$ is just the so-called multiplicity of the linear system at $x$, which is defined by

$$
\nu(|L|, x):=\min \{\nu(E, x), E \in|L|\} .
$$

If a modification $\mu: \widetilde{X} \rightarrow X$ is chosen such that $\mu^{\star}|L|=|M|+F$, where $M$ has no base-point and $F$ is an effective divisor, then $\mu^{\star} T_{|L|}=T_{\mu^{\star}|L|}=T_{|M|}+F$ where $T_{|M|}$ is smooth since $|M|$ is generated by global sections. The so-called moving self-intersection of $L$, which is by definition $L^{[n]}:=M^{n}$, is thus also equal to $\int_{X}\left(T_{|L|}\right)_{a c}^{n}$. 
When $L$ is a big line bundle, we get, for each $k>0$ big enough, a positive current $T_{k}:=\frac{1}{k} T_{|k L|}$ in $c_{1}(L)$. A result of Fujita (cf. [9]) claims that the volume $v(L)$ is the limit of $\frac{1}{k^{n}}(k L)^{[n]}$, thus we have $v(L)=\lim _{k \rightarrow+\infty} \int_{X} T_{k, a c}^{n}$.

Finally, if $T_{\min }$ is a positive current with minimal singularities in $c_{1}(L)$, we can choose a singular Hermitian metric $h_{\min }$ on $L$ whose curvature current is $T_{\min }$ (by Section 2.4). If $L$ is still big and if for each $k$ we choose the basis of $H^{0}(k L)$ to be orthonormal with respect to $h_{\mathrm{min}}^{\otimes k}$, then it can be shown that $T_{k} \rightarrow T_{\min }$, and we will see in 5.2 that $\nu\left(T_{k}, x\right)=\frac{1}{k} \nu(|k L|, x)$ converges to $\nu\left(T_{\min }, x\right)=\nu\left(c_{1}(L), x\right)$. In some sense, the family $T_{k}$ deriving from $|k L|$ is cofinite $\left(c_{1}(L)^{+}, \preceq\right)$.

It should however be stressed that $T_{|k L|}$ will in general not be a Kähler current, even if $L$ is big. Indeed, consider the pull-back $L=\mu^{\star} A$ of some ample line bundle $A$ by a blow-up $\mu$. Then $k L$ will be generated by global sections for $k$ big enough, and $T_{|k L|}$ is thus smooth for such a $k$, but not a Kähler current, since $L$ is not ample and a smooth Kähler current is just a Kähler form.

Conversely, to go from currents to sections is the job of the $L^{2}$ estimates for the $\bar{\partial}$ operator, e.g. in the form of Nadel's vanishing theorem. Recall that the multiplier ideal sheaf $\mathcal{I}(T)$ of a closed almost positive $(1,1)$-current $T$ is defined locally as follows: write $T=d d^{c} \varphi$ locally at some $x$. Then the stalk $\mathcal{I}(T)_{x}$ is the set of germs of holomorphic functions at $x$ such that $|f|^{2} \mathrm{e}^{-2 \varphi}$ is locally integrable at $x$. Then Nadel's vanishing states that if $T$ is a Kähler current in the first Chern class $c_{1}(L)$ of a line bundle $L$, then $H^{q}\left(X, \mathcal{O}\left(K_{X}+L\right) \otimes \mathcal{I}(T)\right)=0$ for every $q>0$. In particular, if $V(T)$ denotes the scheme $V(\mathcal{I}(T))$, then the restriction map

$$
H^{0}\left(X, \mathcal{O}_{X}\left(K_{X}+L\right)\right) \rightarrow H^{0}\left(V(T), \mathcal{O}_{V(T)}\left(K_{X}+L\right)\right)
$$

is surjective. This gives a tool to prove the generation of jets at some points, using the following lemma (cf. [9]):

LEMMA 5.1 (Skoda's lemma). - If $\nu(T, x)<1$, then $\mathcal{I}(T)_{x}=\mathcal{O}_{x}$. If $\nu(T, x) \geqslant n+s$, we have $\mathcal{I}(T)_{x} \subset \mathcal{M}_{x}^{s+1}$.

To illustrate how this works, let us prove the following algebraic characterization of the nonKähler locus:

Proposition 5.2. - If $L$ is a big line bundle, then the non-Kähler locus $E_{n K}\left(c_{1}(L)\right)$ is the intersection of the non-finite loci $\Sigma_{k}$ of the rational maps $\phi_{|k L|}$, defined as the union of the reduced base locus $B_{|k L|}$ and the set of $x \in X-B_{|k L|}$ such that the fiber through $x$ $\phi_{|k L|}^{-1}\left(\phi_{|k L|}(x)\right)$ is positive-dimensional somewhere.

Proof. - If $x_{1}, \ldots, x_{r} \in X$ lie outside $E_{n K}\left(c_{1}(L)\right)$, then we can find a Kähler current $T \in c_{1}(L)$ with analytic singularities such that each $x_{j}$ lies outside the singular locus of $T$. The latter being closed, there exists a neighbourhood $U_{j}$ of $x_{j}$ such that $\nu(T, z)=0$ for every $z \in U_{j}$. We artificially force an isolated pole at each $x_{j}$ by setting

$$
\widetilde{T}=T+\sum_{1 \leqslant j \leqslant r} d d^{c}\left(\varepsilon \theta_{j}(z) \log \left|z-x_{j}\right|\right)
$$

where $\theta_{j}$ is a smooth cut-off function near $x_{j}$, and $\varepsilon>0$ is so small that $\widetilde{T}$ is still Kähler. We have $\nu\left(\widetilde{T}, x_{j}\right)=\varepsilon$, whereas $\nu(\widetilde{T}, z)$ is still zero for every $z \neq x_{j}$ in $U_{j}$. We now choose some smooth form $\tau$ in $c_{1}\left(K_{X}\right)$, and consider the current $T_{k}:=k \widetilde{T}-\tau$. It lies in the first Chern class of $L_{k}:=k L-K_{X}$, and is certainly still Kähler for $k$ big enough. We also have $\nu\left(T_{k}, z\right)=0$ for every $z \neq x_{j}$ close to $x_{j}$, and $\nu\left(T_{k}, x_{j}\right)=k \varepsilon$. Given $s_{1}, \ldots, s_{r}$, we see that, for $k$ big enough, 
each $x_{j}$ will be isolated in $E_{1}\left(T_{k}\right)$, whereas $\mathcal{I}\left(T_{k}\right)_{x_{j}} \subset \mathcal{M}_{x_{j}}^{s_{j}+1}$, using Skoda's lemma. Nadel's vanishing then implies that the global sections of $k L$ generate $s_{j}$-jets at $x_{j}$ for every $j$. This implies that the non-finite locus $\Sigma_{k}$ is contained in $E_{n K}\left(c_{1}(L)\right)$.

To prove the converse inclusion, we have to find for each $m$ a Kähler current $T_{m}$ in $c_{1}(L)$ with $E_{+}\left(T_{m}\right) \subset \Sigma_{m}$. To do this, we copy the proof of Proposition 7.2 in [6].

LEMMA 5.3. - If $L$ is any line bundle such that the non-finite locus $\Sigma_{m}$ of $m L$ is distinct from $X$ for some $m$, then, for every line bundle $G$, the base locus of $|k L-G|$ is contained in $\Sigma_{m}$ for $k$ big enough.

We then take $G$ to be ample, and set $T_{m}:=\frac{1}{k}\left(T_{|k L-G|}+\omega\right)$ with $k$ big enough so that $B_{|k L-G|} \subset \Sigma_{m}$ and $\omega$ a Kähler form in $c_{1}(G)$.

To prove Lemma 5.3, note that $|m L|$ is not empty, so we can select a modification $\mu: \widetilde{X} \rightarrow X$ such that $\mu^{\star}|m L|=|\widetilde{L}|+F$, where $|\widetilde{L}|$ is base-point free. It is immediate to check that it is enough to prove the lemma for $\widetilde{L}$, so we can assume from the beginning that $L$ is base-point free, with $m=1$. We set $\phi:=\phi_{|L|}: X \rightarrow \mathbf{P}^{N}$ and $\Sigma:=\Sigma_{1}$. Upon adding a sufficiently ample line bundle to $G$, it is also clear that we may assume $G$ to be very ample. If $x \in X$ lies outside $\Sigma$, the fiber $\phi^{-1}(\phi(x))$ is a finite set, so we can find a divisor $D \in|G|$ which does not meet it. Therefore we have $\phi(x) \in \mathbf{P}^{N}-\phi(D)$, so that for $k$ big enough there exists $H \in\left|\mathcal{O}_{\mathbf{P}^{N}}(k)\right|$ with $H \geqslant \phi_{\star} D$ which does not pass through $\phi(x)$. The effective divisor $\phi^{\star} H-D$ is then an element of $|k L-G|$ which does not pass through $x$. The upshot is: for every $x \in X$ outside $\Sigma$, we have $x \in X-B_{|k L-G|}$ for $k$ big enough. By Nötherian induction, we therefore find $k$ big enough such that $B_{|k L-G|}$ is contained in $\Sigma$, as was to be shown.

\subsection{Minimal Lelong numbers}

When $L$ is a big $\mathbf{R}$-divisor, we denote by $L_{k}:=\lfloor k L\rfloor$ the round-down of $k L$, and by $R_{k}:=k L-L_{k}$ the fractional part of $k L$. We then consider the sequence $\frac{1}{k} \nu\left(\left|L_{k}\right|, x\right)$. It is easily seen to be subadditive, and therefore $\nu(|| L||, x):=\lim _{k \rightarrow+\infty} \frac{1}{k} \nu(|k L|, x)$ exists. We then prove the following

THEOREM 5.4. - If L is a big $\mathbf{R}$-divisor on $X$ and $\alpha:=\{L\} \in N S(X)_{\mathbf{R}}$, then

$$
\nu(\alpha, x)=\nu(\|L\|, x)
$$

for every $x \in X$.

Proof. - Let $L=\sum a_{j} D_{j}$ be the decomposition of $L$ into its prime components. We choose arbitrary smooth forms $\eta_{j}$ in $\left\{D_{j}\right\}$, and denote by $\tau_{k}:=\sum\left(k a_{j}-\left\lfloor k a_{j}\right\rfloor\right) \eta_{j}$ the corresponding smooth form in $\left\{R_{k}\right\}$. Since $\tau_{k}$ has bounded coefficients, we can choose a fixed Kähler form $\omega$ such that $-\omega \leqslant \tau_{k} \leqslant \omega$ for every $k$. If $E$ is an effective divisor in $\left|L_{k}\right|$, then $1 / k\left([E]+\tau_{k}\right)$ is a current in $\alpha[-1 / k \omega]$, therefore $\frac{1}{k} \nu(E, x) \geqslant \nu\left(T_{\min , 1 / k}, x\right)$, where $T_{\min , 1 / k}$ is a current with minimal singularities in $\alpha[-1 / k \omega]$, and this yields

$$
\lim _{k \rightarrow \infty} \frac{1}{k} \nu\left(\left|L_{k}\right|, x\right) \geqslant \lim _{k \rightarrow \infty} \nu\left(T_{\min , 1 / k}, x\right)=\nu(\alpha, x) .
$$

In the other direction, we use a related argument in [9], Theorem 1.11. The Ohsawa-TakegoshiManivel $L^{2}$ extension theorem says in particular that if we are given a Hermitian line bundle $\left(A, h_{A}\right)$ with sufficiently positive curvature form, then for every pseudo-effective line bundle $G$ 
and every singular Hermitian metric $h$ on $G$ with positive curvature current $T \in c_{1}(G)$ and every $x \in X$, the evaluation map

$$
H^{0}(X, \mathcal{O}(G+A) \otimes \mathcal{I}(T)) \rightarrow \mathcal{O}_{x}(G+A) \otimes \mathcal{I}(T)_{x}
$$

is surjective, with an $L^{2}$ estimate independent of $(G, h)$ and $x \in X$.

We now fix a Hermitian line bundle $\left(A, h_{A}\right)$ with a sufficiently positive curvature form $\omega_{A}$ to satisfy the Ohsawa-Takegoshi theorem. We select a positive current with minimal singularities $T_{\min }$ in $\alpha$, and also a Kähler current $T$ in $\alpha$, which is big by assumption; we can then find almost pluri-subharmonic functions $\varphi_{\min }$ and $\varphi$ on $X$ such that $T_{\min }-d d^{c} \varphi_{\min }$ and $T-d d^{c} \varphi$ are smooth. We set $G_{k}:=L_{k}-A=k L-R_{k}-A=\left(k-k_{0}\right) L+\left(k_{0} L-R_{k}-A\right)$, and fix $k_{0}$ big enough so that $k_{0} T-\omega-\omega_{A}$ is a Kähler current. For $k \geqslant k_{0}$, the current $T_{k}:=\left(k-k_{0}\right) T_{\min }+\left(k_{0} T-\tau_{k}-\omega_{A}\right)$ is then a positive current in $c_{1}\left(G_{k}\right)$, thus we can choose for each $k$ a smooth Hermitian metric $h_{k}$ on $G_{k}$ such that $T_{k}$ is the curvature current of the singular Hermitian metric $\exp \left(-2\left(k-k_{0}\right) \varphi_{\min }-2 k_{0} \varphi\right) h_{k}$. Applying the Ohsawa-Takegoshi to $G_{k}$ equipped with this singular Hermitian metric, we thus get a section $\sigma \in H^{0}\left(X, L_{k}\right)$ such that

$$
h_{k}(\sigma(x)) \exp \left(-2\left(k-k_{0}\right) \varphi_{\min }(x)-2 \varphi(x)\right)=1
$$

and

$$
\int_{X} h_{k}(\sigma) \exp \left(-2\left(k-k_{0}\right) \varphi_{\min }-2 \varphi\right) d V \leqslant C_{1},
$$

where $C_{1}$ does not depend on $k$ and $x$. If we choose a basis $\sigma_{1}, \ldots, \sigma_{l}$ of $H^{0}\left(X, L_{k}\right)$, we infer from this that

$$
\begin{aligned}
\varphi_{\min }(x)+\frac{1}{k-k_{0}} \varphi(x) & =\frac{1}{2\left(k-k_{0}\right)} \log h_{k}(\sigma(x)) \\
& \leqslant \frac{1}{2\left(k-k_{0}\right)} \log \sum h_{k}\left(\sigma_{j}(x)\right)+C_{2},
\end{aligned}
$$

where $C_{2}$ does not depend on $x$. The latter inequality comes from the bound on the $L^{2}$ norm of $\sigma$, since the $L^{2}$ norm dominates the $L^{\infty}$ norm. Therefore

$$
\frac{1}{k-k_{0}} \nu\left(\left|L_{k}\right|, x\right) \leqslant \nu\left(\varphi_{\min }, x\right)+\frac{C_{3}}{k-k_{0}}
$$

where $C_{3}$ is a bound on the Lelong numbers of $T$. If we let $k \rightarrow \infty$ in the last inequality, we get $\nu(\|L\|, x) \leqslant \nu(\alpha, x)$ as desired.

\subsection{Zariski decompositions of a divisor}

The usual setting for the problem of Zariski decompositions is the following: let $X$ be a projective manifold, and $L$ a divisor on it. One asks when it is possible to find two R-divisors $P$ and $N$ such that:

(i) $L=P+N$,

(ii) $P$ is nef,

(iii) $N$ is effective,

(iv) $H^{0}(X, k L)=H^{0}(X,\lfloor k P\rfloor)$ for all $k>0$, where the round-down $\lfloor F\rfloor$ of an $\mathbf{R}$-divisor $F$ is defined coefficient-wise. 
This can of course happen only if $L$ is already pseudo-effective. When this is possible, one says that $L$ admits a Zariski decomposition (over $\mathbf{R}$ or $\mathbf{Q}$, depending whether the divisors are real or rational). We want to show that, for a big divisor $L$, this can be read off the negative part $N(\{L\})$.

THEOREM 5.5. - Let L be a big divisor on $X$, and let

$$
N(L):=N(\{L\}) \text { and } P(L):=L-N(L) .
$$

Then $L=P(L)+N(L)$ is the unique decomposition $L=P+N$ into a modified nef $\mathbf{R}$-divisor $P$ and an effective $\mathbf{R}$-divisor $N$ such that the canonical inclusion $H^{0}(\lfloor k P\rfloor) \rightarrow H^{0}(k L)$ is an isomorphism for each $k>0$.

Proof. - First, we have to check that $H^{0}(X, k L)=H^{0}(X,\lfloor k P(L)\rfloor)$. If $E$ is an effective divisor in the linear system $|k L|$, we have to see that $E \geqslant\lceil k N(L)\rceil$. But $\frac{1}{k}[E]$ is a positive current in $\{L\}$, thus $E \geqslant k N(L)$, and so $E \geqslant\lceil k N(L)\rceil$ since $E$ has integer coefficients.

Conversely, assume that $L=P+N$ is a decomposition as in Theorem 5.5. We have to show that $N=N(L)$, i.e. $\nu(\{L\}, D)=\nu(N, D)$ for every prime $D$. In view of Theorem 5.4, this will be a consequence of the following

Lemma 5.6. - Suppose that a big divisor $L$ writes $L=P+N$, where $P$ is an $\mathbf{R}$-divisor and $N$ is an effective $\mathbf{R}$-divisor such that $H^{0}(X, k L)=H^{0}(X,\lfloor k P\rfloor)$ for every $k>0$. Then we have:

(i) If $P$ is nef, then $\nu(\|L\|, x)=\nu(N, x)$ for every $x \in X$.

(ii) If $P$ is modified nef, then $\nu(\|L\|, D)=\nu(N, D)$ for every prime $D$.

Proof. - The assumption $H^{0}(X, k L)=H^{0}(X,\lfloor k P\rfloor)$ means precisely that for every $E \in|k L|$ we have $E \geqslant\lceil k N\rceil$, thus $\nu(|k L|, x) \geqslant \sum \frac{\left\lceil k a_{j}\right\rceil}{k} \nu\left(D_{j}, x\right)$ if we write $N=\sum a_{j} D_{j}$. We deduce from this the inequality $\lim _{k \rightarrow \infty} \frac{1}{k} \nu(|k L|, x) \geqslant \sum a_{j} \nu\left(D_{j}, x\right)=\nu(N, x)$. To get the converse inequalities, notice that

$$
\nu(|k L|, x) \leqslant \nu\left(\left|P_{k}\right|, x\right)+\nu(k N, x)
$$

with $P_{k}:=\lfloor k P\rfloor$ as before; dividing this out by $k$ and letting $k \rightarrow+\infty$, we deduce

$$
\lim _{k \rightarrow \infty} \frac{1}{k} \nu(|k L|, x) \leqslant \lim _{k \rightarrow \infty} \frac{1}{k} \nu(k N, x)=\nu(N, x)
$$

when $P$ is nef, since $\nu(\{P\}, x)=\lim _{k \rightarrow \infty} \frac{1}{k} \nu\left(P_{k}, x\right)$ is then always zero, and similarly with $D$ in place of $x$ when $P$ is modified nef (remark that $P$ is big because $L$ is). This concludes the proof of Theorem 5.5.

COROLlARY 5.7 (Cutkosky's criterion). - Let L be a big divisor on $X$, and assume that $\nu(\{L\}, D)$ is irrational for some irreducible divisor $D$. Then there cannot exist a modification $\mu: \widetilde{X} \rightarrow X$ such that $\mu^{\star} L$ admits a Zariski decomposition over $\mathbf{Q}$.

Proof. - If a modification $\mu$ as stated exists, then the negative part $N\left(\mu^{\star} L\right)$ has to be rational by Theorem 5.5, and we get a contradiction using the following easy

LEMMA 5.8. - Let $\alpha$ be a pseudo-effective class on $X$, and let $\mu: \widetilde{X} \rightarrow X$ be a modification. Then we have

$$
N(\alpha)=\mu_{\star} N\left(\mu^{\star} \alpha\right)
$$

Proof. - Very easily checked using that a modification is an isomorphism in codimension 1. 


\subsubsection{An example of Cutkosky}

We propose to analyze in our setting an example due to S.D. Cutkosky [3] of a big line bundle $L$ on a 3 -fold $X$ whose divisorial Zariski decomposition is not rational, but whose Zariski projection $Z(\{L\})$ is nef. We start from any projective manifold $Y$ for which $\mathcal{N}_{Y}=\mathcal{E}_{Y}$. Thus $Y$ might be a smooth curve or any manifold with nef tangent bundle (cf. [8]). We pick two very ample divisors $D$ and $H$ on $Y$, and consider $X:=\mathbf{P}(\mathcal{O}(D) \oplus \mathcal{O}(-H))$, with its canonical projection $\pi: X \rightarrow Y$. If we denote by $L:=\mathcal{O}(1)$ the canonical relatively ample line bundle on $X$, then it is well known that

$$
H^{1,1}(X, \mathbf{R})=\pi^{\star} H^{1,1}(Y, \mathbf{R}) \oplus \mathbf{R} L .
$$

Since $D$ is ample, $L$ is big, but it will not be nef since $-H$ is not. We are first interested in the divisorial Zariski decomposition of $L$. We have a hypersurface $E:=\mathbf{P}(\mathcal{O}(-H)) \subset X$, and since $D$ has a section, we see that $E+\pi^{\star} D \in|L|$. Therefore we get $N(L) \leqslant N\left(\pi^{\star} D\right)+E$; but $\pi^{\star} D$ is nef, so has $N\left(\pi^{\star} D\right)=0$, and we deduce $N(L) \leqslant E$. Consequently, $N(L)=\mu_{L} E$ for some $0 \leqslant \mu_{L} \leqslant 1$, and $L=Z(L)+\mu_{L} E$. We claim that

$$
\mu_{L}=\min \left\{t>0,(L-t E)_{\mid E} \in \mathcal{N}_{E}\right\} .
$$

First, we have $L-t E=\pi^{\star} D+(1-t) E$, and since $\pi^{\star} D$ is nef, we get that the non-nef locus $E_{n n}(L-t E)$ is contained in $E$ for $0<t<1$. Therefore $L-t E \in \mathcal{N}_{X}$ iff $(L-t E)_{\mid E} \in \mathcal{N}_{E}$. If this is the case, we have $N(L) \leqslant N(L-t E)+t E=t E$, and thus $t \geqslant \mu_{L}$. Conversely, since $L-\mu_{L} E=Z(L)$ lies in $\mathcal{M N}$, we get that $Z(L)_{\mid E} \in \mathcal{E}_{E}=\mathcal{N}_{E}$ by Proposition 2.4 (since $E$ is isomorphic to $Y$ via $\pi$ ), and we deduce the equality. Now, notice that the projection $\pi$ induces an isomorphism $E \rightarrow Y$ such that $L$ becomes $-H$ and thus $E_{\mid E}$ becomes $-D-H$. The condition $(L-t E)_{\mid E} \in \mathcal{N}_{E}$ is turned into $-H+t(D+H) \in \mathcal{N}_{Y}$, and we get in the end

$$
\mu_{L}=\min \left\{t>0,-H+t(D+H) \in \mathcal{N}_{Y}\right\}
$$

The picture can be made more precise:

Proposition 5.9. - (i) The nef cone $\mathcal{N}_{X}$ is generated by $\pi^{\star} \mathcal{N}_{Y}$ and $L+\pi^{\star} H$.

(ii) The pseudo-effective cone $\mathcal{E}_{X}$ is generated by $\pi^{\star} \mathcal{N}_{Y}$ and by $E$.

(iii) The only exceptional divisor on $X$ is $E$, and the modified Kähler cone coincides with the Kähler cone. The Zariski projection $Z(\alpha)$ of a pseudo-effective class $\alpha$ is thus the projection of $\alpha$ on $\mathcal{N}_{X}$ parallel to $\mathbf{R}_{+} E$.

Proof. - Given line bundles $L_{1}, \ldots, L_{r}$ on a compact Kähler manifold $Y$, a class $\alpha=\pi^{\star} \beta$ over

$$
X:=\mathbf{P}\left(L_{1} \oplus \cdots \oplus L_{r}\right)
$$

is nef (respectively pseudo-effective) iff $\beta$ is. A class $\alpha=\mathcal{O}(1)+\pi^{\star} \beta$ is nef iff $\beta+L_{j}$ is nef for all $j$, and $\alpha$ is big iff the convex cone generated by $\beta+L_{1}, \ldots, \beta+L_{r}$ meets the big cone of $Y$, which condition is equivalent (by homogeneity) to: $\beta+\operatorname{conv}\left(L_{1}, \ldots, L_{r}\right)$ meets the big cone; finally $\alpha$ is pseudo-effective iff $\beta+\operatorname{conv}\left(L_{1}, \ldots, L_{r}\right)$ meets $\mathcal{E}_{Y}$. In our case $\alpha=\pi^{\star} \beta+L$ is thus nef iff $\beta-H$ is nef, and $\alpha$ is pseudo-effective iff $\alpha+[-H, D]$ meets $\mathcal{N}_{Y}$. The latter condition is clearly equivalent to $\alpha-D \in \mathcal{N}_{Y}$. Now an arbitrary class $\alpha$ on $X$ uniquely writes $\alpha=t L+\pi^{\star} \beta$. If $\alpha$ is pseudo-effective, then $t \geqslant 0$ (since $L$ is relatively ample); if $t=0$, then $\alpha \in \pi^{\star} \mathcal{N}_{Y}$. Otherwise, we may assume by homogeneity that $t=1$, and thus (i) and (ii) follow from the above discussion. 
By (ii), a pseudo-effective class $\alpha$ writes $\pi^{\star} \beta+t E$ with $\beta$ nef. Therefore we get $N(\alpha) \leqslant t E$, and $E$ is thus the only exceptional divisor on $X$. In fact, we even have $E_{n n}(\alpha) \subset E$, and thus $\alpha$ is nef iff $\alpha_{\mid E}$ is nef. In particular, we see that $\mathcal{M K}=\mathcal{K}$ as desired (use Proposition 2.4 again).

We now assume that $Y$ is a surface. The assumption $\mathcal{N}_{Y}=\mathcal{E}_{Y}$ implies that $\mathcal{N}_{Y}=\overline{\mathcal{P}}_{Y}=\mathcal{E}_{Y}$, and $\mu_{L}$ is none but the least of the two roots of the quadratic polynomial in $t(-H+t(D+H))^{2}$; it will thus be irrational for most choices of $H$ and $D$ (on, say, an abelian surface). This already yields that the divisorial Zariski decomposition of the rational class $c_{1}(L)$ will not be rational in general, that is, the analogue of Corollary 4.11 is not true in general on a 3 -fold.

Since $Z(L)$ is nef, the volume of $L$ is just $v(Z(L))=Z(L)^{3}$, with

$$
Z(L)=\left(1-\mu_{L}\right) L+\mu_{L} \pi^{\star} D .
$$

The cubic intersection form is explicit on $H^{1,1}(X, \mathbf{R})$ from the relations

$$
L^{3}-\pi^{\star}(D-H) \cdot L^{2}-D \cdot H \cdot L=0
$$

and $\pi_{\star} L=1, \pi_{\star} L^{2}=D-H$, thus we can check that $v(L)$ is an explicit polynomial of degree 3 in $\mu_{L}$ which is also irrational for most choices of $D$ and $H$. We conclude: there exists a big line bundle on a projective 3 -fold with an irrational volume, by contrast with Proposition 4.12.

\section{Appendix A. Nakayama's algebraic approach}

\section{A.1. Algebraic minimal multiplicities}

In this appendix, we will briefly survey Nakayama's algebraic approach to the divisorial Zariski decomposition. Consider a projective manifold $X$, and a big $\mathbf{R}$-divisor $B$ on it. Denote by $|B|$ the set of effective $\mathbf{R}$-divisors which are linearly equivalent to $B$, in the sense that their round-downs are linearly equivalent and their fractional part coincide, and by $|B|_{\mathbf{Q}}:=\bigcup_{k>0} \frac{1}{k}|k B|$ the set of effective $\mathbf{R}$-divisors which are $\mathbf{Q}$-linearly equivalent to $B$. Nakayama then sets for every $x \in X$ :

$$
\sigma_{x}(B):=\inf \left\{\nu(D, x), D \in|B|_{\mathbf{Q}}\right\} .
$$

This is clearly homogeneous and convex with respect to $B$, and is zero for every $x \in X$ as soon as $B$ is ample. Note that it is none but what we denoted by $\nu(\|B\|, x)$ in 5.2. Now take $B$ as before, and fix an ample R-divisor $A$ such that $B-A=: C$ is effective. If $D$ is numerically equivalent to $B$ and $\varepsilon>0$ is given, we have $(1+\varepsilon) B=(B-D+\varepsilon A)+D+\varepsilon C$, where $B-D+\varepsilon A$ is ample, and thus $(1+\varepsilon) \sigma_{x}(B) \leqslant \sigma_{x}(D)+\varepsilon \sigma_{x}(C)$ by homogeneity and convexity. Letting $\varepsilon$ go to zero, we get $\sigma_{x}(B) \leqslant \sigma_{x}(D)$, which shows by symmetry that $\sigma_{x}(B)=\inf \left\{\nu(D, x), D \in|B|_{\mathbf{R}}\right\}$, where $|B|_{\mathbf{R}}$ is the set of effective $\mathbf{R}$-divisors numerically equivalent to $B$. This argument, due to Nakayama, shows that the minimal multiplicity $\sigma_{x}(B)$ of the big $\mathbf{R}$-divisor $B$ only depends on its numerical class, and is computed as a $\operatorname{limit}_{\lim _{k \rightarrow+\infty}} \nu\left(B_{k}, x\right)$ for some sequence $B_{k} \in|B|_{\mathbf{R}}$. But the latter set is non-compact, and admits as a natural compactification the set of all closed positive currents cohomologous to $B$. The limit object which computes $\sigma_{x}(B)$ is then a closed positive current with minimal singularities, and $\sigma_{x}(B)$ is just the minimal multiplicity $\nu(\alpha, x)$ we have defined, if $\alpha:=\{B\}$ is the cohomology class of $B-$ this is the content of our Theorem 5.4.

Going back to the algebraic setting, Nakayama defines $\sigma_{x}(D)$ for an arbitrary pseudo-effective R-divisor $D$ as the limit of $\sigma_{x}(D+\varepsilon A)$, where $A$ is ample. Each of the formal properties of the 
minimal multiplicities (continuity, finiteness of the divisorial part of the non-nef locus, ...) are established by Nakayama using divisors only, and then going to the limit. The crucial point, as in the argument above, is that ampleness is a numerical property.

We will now present an algebraic characterization of the non-nef locus given in [16]. Just as a pseudo-effective class is the numerical analogue of an effective divisor, a nef class is the analogue of a base-point free divisor. The non-nef locus is thus a "numerical base locus", and Nakayama relates it to the usual base loci by proving the

THEOREM A.1. - There exists a sufficiently ample divisor $A$ on $X$ such that: if $D$ is a pseudoeffective divisor, then its non-nef locus is the union of all the base loci of $|k D+A|$ for $k \geqslant 0$.

Nakayama's proof uses the Kawamata-Viehweg vanishing theorem, but is most easily explained using Nadel's formulation: take $A$ to be any ample divisor such that $A-K_{X}-n H$ is still ample, where $H$ is very ample. Then one can find, for every $x \in X$, a Kähler current $T$ in the class of $A-K_{X}$ with an isolated singularity at $x$ such that $\nu(T, x)=n$. Suppose now that $x$ is a nef point of $D$. Then one can find currents in its class which are smooth near $x$ and with an arbitrarily small negative part; using the current $T$ above, we can thus find for every $k>0$ a Kähler current in $k D+A-K_{X}$ with an isolated singularity at $x$ of multiplicity $n$. Skoda's lemma and Nadel's vanishing then imply that $k D+A$ is free at $x$.

\section{A.2. Two counter-examples}

We now present two constructions from [16], based on the same ideas as Cutkosky's Example 5.3.1, which provide counter-examples to natural questions concerning Zariski decompositions.

The first case is a discontinuous minimal multiplicity (cf. Proposition 3.5): let $L$ be a nef line bundle squaring to zero over a surface $Y$ whose nef cone coincides with its positive cone (such as an abelian surface), and set $\pi: X=\mathbf{P}(\mathcal{O} \oplus \mathcal{O}(-L)) \rightarrow Y$, and $D_{0}:=\mathbf{P}(\mathcal{O}(-L)) \subset X$. As in 5.3.1, one checks that if $\alpha=\pi^{\star} \beta+H$ is a pseudo-effective class on $X$, then $\nu\left(\alpha, D_{0}\right)$ is the least real number $t \geqslant 0$ such that $\beta-(1-t) L$ is nef. If we choose $\beta \in \partial \mathcal{P}$ and $\varepsilon>0$, then $(\beta-\varepsilon L)^{2}=-2 \varepsilon \beta \cdot L \leqslant 0$, and this is zero iff $\beta$ is proportional to $L$, since $L^{\perp}$ meets $\partial \mathcal{P}$ along $\mathbf{R} L$ by the non-degeneracy of the intersection form. It follows that $\nu\left(\pi^{\star} L+H, D_{0}\right)=0$, whereas $\nu\left(\pi^{\star} \beta+H, D_{0}\right)=1$ as soon as $\beta \in \partial \mathcal{P}$ is not proportional to $L$. In particular, $\alpha \mapsto \nu\left(\alpha, D_{0}\right)$ is not continuous at $\pi^{\star} L+H$.

The second example is much more important but also much more involved, and we will only describe it. It yields a nef and big line bundle $L$ on a 4 -fold $X$ for which no modification $\mu: \widetilde{X} \rightarrow X$ can be found so that $Z\left(\mu^{\star} L\right)$ be nef. In other words, it shows the non-existence of a Zariski decomposition in general, even in its most optimistic form. To get this, one starts again from a surface $Y$ whose nef cone is the closure of $\mathcal{P}$, and which also admits two ample line bundles $A_{1}$ and $A_{2}$ whose cohomology classes are not proportional (for instance an abelian surface with Picard number at least 2). Then one considers $\pi: X=\mathbf{P}\left(\mathcal{O} \oplus \mathcal{O}\left(A_{1}\right) \oplus \mathcal{O}\left(A_{2}\right)\right) \rightarrow Y$, $H:=\mathcal{O}(1)$ and a big class $\alpha=\pi^{\star} \beta+H$ which satisfies the following conditions:

(i) $\beta$ is not nef.

(ii) $\beta+A_{1}$ and $\beta+A_{2}$ are nef.

As in 5.3.1, one then checks that $\alpha$ is nef in codimension 1 but not nef. Its non-nef locus is $V=D_{1} \cap D_{2}$, with $D_{1}=\mathbf{P}\left(\mathcal{O} \oplus \mathcal{O}\left(A_{2}\right)\right) \subset X$ and $D_{2}=\mathbf{P}\left(\mathcal{O} \oplus \mathcal{O}\left(A_{1}\right)\right) \subset X$. The minimal multiplicity $\nu(\alpha, x)=\nu$ is furthermore constant for $x \in V$, equal to the least real number $t \geqslant 0$ such that $\beta+t\left[a_{1}, a_{2}\right]$ meets $\overline{\mathcal{P}}$. Let us add the following third requirement:

(iii) $\beta+\nu\left[a_{1}, a_{2}\right]$ is tangent to $\overline{\mathcal{P}}$ at $\beta+\nu x$ for some $\left.x \in\right] a_{1}, a_{2}[$.

In order to get a Zariski decomposition of $\alpha$, the idea is to successively blow up the non-nef loci and subtract the divisorial part at each step. Thus we set $X_{0}:=X, E_{0}:=D_{1}, G_{0}:=D_{2}$ 
and $V_{0}:=V$. Consider the blow-up $\pi_{1}: X_{1} \rightarrow X_{0}$ along $V_{0}$ with exceptional divisor $E_{1}$. Denote by $G_{1}$ the strict transform of $G_{0}$, which is a smooth hypersurface of $X_{1}$ meeting $E_{1}$ transversally along a surface $V_{1}$ isomorphic to $V$ under the projection map. Define inductively $\pi_{n}: X_{n} \rightarrow X_{n-1}$ as the blow-up along $V_{n-1}$, with exceptional divisor $E_{n}$, and let $G_{n}$ be the strict transform of $G_{n-1}$, which cuts $E_{n}$ transversally along a surface $V_{n}$ isomorphic to $V$ by projection. At each step, set $\alpha_{n}:=Z\left(\mu_{n}^{\star} \alpha_{n-1}\right)$, which is also equal to $\pi_{n}^{\star} \alpha_{n-1}-$ $\nu\left(\alpha_{n-1}, V_{n-1}\right)\left\{E_{n}\right\}$ thanks to the relation

$$
\nu\left(\pi_{n}^{\star} \alpha_{n-1}, E_{n}\right)=\nu\left(\alpha_{n-1}, V_{n-1}\right)
$$

(this is easy to obtain in the algebraic setting, but can also be proved in the more general analytic setting). In other words, $\alpha_{n}$ is the residual part of $\pi_{n}^{\star} \alpha_{n-1}$ once its negative divisorial part has been subtracted. Then the main fact proved in [16] is that $V_{n}$ is contained in the non-nef locus of $\alpha_{n}$ for every $n$, i.e. that $\nu_{n}:=\nu\left(\alpha_{n}, V_{n}\right)$ is positive.

We will not reproduce the proof of this fact, which is long and technical, but we will give the proof of the following criterion from [16] which enables to conclude that there exists NO modification $\mu: \widetilde{X} \rightarrow X$ such that $Z\left(\mu^{\star} \alpha\right)$ is nef.

LEMMA A.2. - Let $\pi_{n}: X_{n} \rightarrow X_{n-1}$ be a sequence of blow-ups with smooth 2-codimensional centre $V_{n-1} \subset X_{n-1}$ and exceptional divisor $E_{n} \subset X_{n}$, and let $\alpha_{0}$ be a big class on $X_{0}$ which is nef in codimension 1 , and such that:

(i) $V_{n} \subset E_{n}$ for each $n$.

(ii) $V_{n}$ is contained in the non-nef locus of the strict transform $\alpha_{n} \in H^{1,1}\left(X_{n}, \mathbf{R}\right)$ of $\alpha_{0}$ under $\pi_{n}$.

Then there exists no modification $\mu_{0}: \widetilde{X}_{0} \rightarrow X_{0}$ such that $Z\left(\mu_{0}^{\star} \alpha_{0}\right)$ is nef.

Proof. - Argue by contradiction. Then one can build two sequences of modifications $\mu_{n}: \widetilde{X}_{n} \rightarrow X_{n}$ and $\widetilde{\pi}_{n}: \widetilde{X}_{n} \rightarrow \widetilde{X}_{n-1}$ such that $\mu_{n-1} \circ \widetilde{\pi}_{n}=\pi_{n} \circ \mu_{n}$. Let $\widetilde{\alpha}_{0}:=\mu_{0}^{\star} \alpha_{0}$ and $\widetilde{\alpha}_{n}$ be the pull-back of $\widetilde{\alpha}_{0}$ under the composed map $\widetilde{X}_{n} \rightarrow \widetilde{X}_{0}$. Then the nefness of $Z\left(\widetilde{\alpha}_{0}\right)$ implies that the Zariski decomposition of $\widetilde{\alpha}_{n}$ is just the pull-back of that of $\widetilde{\alpha}_{0}$ for each $n$. In particular, the number of prime divisors in the negative part of $\widetilde{\alpha}_{n}$ is independent of $n$. On the other hand, we claim that the strict transform of $E_{n} \subset X_{n}$ under $\mu_{n}$ is contained in the non-nef locus of $\widetilde{\alpha}_{n}$, which will imply that the number of components of its negative part is strictly increasing with $n$, and will thus yield a contradiction. Actually, the claim is an immediate consequence of the fact that $E_{n}$ is contained in the non-nef locus of $\pi_{n}^{\star} \alpha_{n-1}$, which in turn follows from the formula

$$
\nu\left(\pi_{n}^{\star} \alpha_{n-1}, E_{n}\right)=\nu\left(\alpha_{n-1}, V_{n-1}\right)
$$

(cf. above).

\section{REFERENCES}

[1] Boucksom S., Le cône kählérien d'une variété hyperkählérienne, C. R. Acad. Sci. Paris Sér. I Math. 333 (2001) 935-938.

[2] BoucKSOM S., On the volume of a line bundle, math.AG/0201031, 2002.

[3] Cutкosky S.D., Zariski decomposition of divisors on algebraic varieties, Duke Math. J. 53 (1986) $149-156$.

[4] Demailly J.-P., Estimations $L^{2}$ pour l'opérateur $\bar{\partial}$ d'un fibré vectoriel holomorphe semi-positif au dessus d'une variété kählérienne complète, Ann. Sci. École Norm. Sup. 15 (1982) 457-511. 
[5] Demailly J.-P., Regularization of closed positive currents and intersection theory, J. Algebraic Geom. 1 (1992) 361-409.

[6] Demailly J.-P., Algebraic criteria for Kobayashi hyperbolic projective varieties and jet differentials, Proc. Symp. Pure Math. 62 (2) (1997).

[7] Demailly J.-P., Paun M., Numerical characterization of the Kähler cone of a compact Kähler manifold, math.AG/0105176, 2001.

[8] Demailly J.-P., Peternell T., Schneider M., Compact complex manifolds with numerically effective tangent bundles, J. Algebraic Geom. 3 (1994) 295-345.

[9] Demailly J.-P., EIN L., LAZARSFELD R., A subadditivity property of multiplier ideals, math.AG/0002035, 2000.

[10] Demailly J.-P., Peternell T., Schneider M., Pseudoeffective line bundles on compact Kähler manifolds, math.AG/0006205, 2000.

[11] Fujita T., On Zariski problem, Proc. Japan Acad., Ser. A 55 (1979) 106-110.

[12] Fujita T., Remarks on quasi-polarized varieties, Nagoya Math. J. 115 (1989) 105-123.

[13] Hartshorne R., Algebraic Geometry, in: GTM, vol. 52, Springer-Verlag, 1977.

[14] HUYBRECHTS D., The Kähler cone of a compact hyperkähler manifold, math.AG/9909109, 1999.

[15] LAmARi A., Courants kählériens et surfaces compactes, Ann. Inst. Fourier 49 (1999) 249-263.

[16] NAKAYAMA N., Zariski decomposition and abundance, preprint RIMS.

[17] PAUn M., Sur l'effectivité numérique des images inverses de fibrés en droites, Math. Ann. 310 (1998) 411-421.

[18] SIU Y.T., Analyticity of sets associated to Lelong numbers and the extension of closed positive currents, Invent. Math. 27 (1974) 53-156.

[19] ZARISKI O., The theorem of Riemann-Roch for high multiples of an effective divisor on an algebraic surface, Ann. of Math. 76 (2) (1962) 560-615.

(Manuscrit reçu le 15 juillet 2002; accepté le 23 avril 2003.)

Sébastien BoucKsom

Institut Fourier,

100 rue des Maths, B.P. 74 ,

38402 Saint-Martin d'Hères Cedex, France

E-mail: sbouckso@free.fr 